\title{
POINTWISE A POSTERIORI ERROR ESTIMATES FOR ELLIPTIC PROBLEMS ON HIGHLY GRADED MESHES
}

\author{
RICARDO H. NOCHETTO
}

\begin{abstract}
Pointwise a posteriori error estimates are derived for linear secondorder elliptic problems over general polygonal domains in $2 \mathrm{D}$. The analysis carries over regardless of convexity, accounting even for slit domains, and applies to highly graded unstructured meshes as well. A key ingredient is a new asymptotic a priori estimate for regularized Green's functions. The estimators lead always to upper bounds for the error in the maximum norm, along with lower bounds under very mild regularity and nondegeneracy assumptions. The effect of both point and line singularities is examined. Three popular local estimators for the energy norm are shown to be equivalent, when suitably interpreted, to those introduced here.
\end{abstract}

\section{INTRODUCTION}

A posteriori error estimators are currently used in a variety of engineering and scientific computations $[4,5,19,21]$. They in fact provide the basis for adaptive mesh refinement and quantitative error control. The ultimate goal is often to equidistribute the local discretization error, typically in the energy norm, via a proper use of information extracted from both the computed solution and data. This can be rephrased in terms of optimizing the computational effort for a given accuracy, which in turn corresponds to avoiding overrefinement. Since the pioneering paper [3], a number of estimators have been proposed and tested for various PDEs [2, 4, 5, 6, 13, 14, 19, 24, 25]. Their success has led to an increasing interest in both applications of existing estimators and development of new ones, possibly for problems of different type or norms other than the energy norm. Pointwise error control, for instance, appears to be crucial for certain nonlinear problems [21], and in any event extremely natural in many practical situations.

Even though asymptotic exactness is a desirable property, it is known to require geometric mesh constraints related to superconvergence that are rarely met in applications. Global equivalence between estimators and the true error is instead a more realistic property to aim for. It guarantees reliability and efficiency of associated mesh refinement algorithms [19]. Equivalence has been derived for the energy norm under the sole assumption of mesh regularity in $[2$,

Received by the editor January 11, 1993.

1991 Mathematics Subject Classification. Primary 65N15, 65N30, 65N50, 35B45.

Key words and phrases. A posteriori error estimates, maximum norm, equivalence, point and line singularities.

This work was partially supported by NSF Grant DMS-9008999. 
$22,24]$, and in [6] with an additional saturation assumption. In all these cases the estimators are computable quantities at the element level, hence inexpensive as compared with the solution process. The constants of equivalence can sometimes be estimated in regard to their dependence on mesh geometry [1]. This provides some quantitative basis for feedback error control in the energy norm. But the possibility of overrefinement is not yet excluded because of the global nature of such a norm.

In this paper we view pointwise a posteriori error estimation in the spirit of $[2,3,6,24]$, namely we fully exploit the residual equation. This enables us to formulate a theory valid for polygonal domains $\Omega \subset \mathbf{R}^{2}$ without restrictions on the size of internal angles or type and strength of singularities. They play indeed a secondary role in our analysis. We consider the following linear elliptic problem:

$$
-\operatorname{div}(\mathbf{A} \cdot \nabla u)=f+\operatorname{div} \mathbf{g} \text { in } \Omega, \quad u=0 \quad \text { on } \partial \Omega,
$$

where both $f$ and $\mathbf{g}$ may be discontinuous but bounded, and $\mathbf{A}$ is a smooth coefficient matrix; precise assumptions and further notation are given in $\S 2$. Jump discontinuities (or rapid variations) of $\mathbf{g}$ may simulate line singularities such as free boundaries (or internal layers), whereas point singularities are typically created by the corners of $\Omega$. We indicate with $u_{\mathscr{g}}$ the piecewise linear finite element solution defined over a highly graded unstructured mesh $\mathscr{T}$ made of triangles $T$ with sides $S \in \mathscr{S}$. We denote by $h_{T}\left(h_{S}\right)$ the size of $T \in \mathscr{T}(S \in \mathscr{S})$, and by $h_{\mathscr{G}}\left(\rho_{\mathscr{T}}\right)$ the biggest (smallest) $h_{T}$. We only assume that $\mathscr{T}$ satisfies the minimum angle condition and the geometric constraint $\rho_{\mathscr{g}} \geq C h_{\mathscr{g}}^{\gamma}$ for some $C>0, \gamma \geq 1$. Suppose for simplicity of exposition that the singularities of $f$ and $\mathbf{g}$ occur across interelement boundaries, and set

$$
\mathscr{C}_{\mathscr{G}}:=\max _{T \in \mathscr{T}}\left(h_{T}^{2}\left\|f+\operatorname{div} \mathbf{A} \cdot \nabla u_{\mathscr{G}}+\operatorname{div} \mathbf{g}\right\|_{L^{\infty}(T)}+h_{T} \|\left[\mathbf{A} \cdot \nabla u_{\mathscr{G}}+\mathbf{g} \rrbracket \|_{L^{\infty}(\partial T)}\right),\right.
$$

which is thus well defined. Hereafter divA indicates the vector whose entries are the divergence of the corresponding columns of $\mathbf{A}$, and $\llbracket \cdot \rrbracket$ stands for the jump operator. It is worth noting that $\mathscr{E}_{9}$ is an inexpensive computable quantity at the element level. In $\S 4$ we prove the existence of constants $C_{1}, C_{2}, h^{*}>0$ independent of $u$ and $\mathscr{T}$ such that

$$
C_{1} \mathscr{\mathscr { E }}_{\mathscr{g}} \leq\left\|u-u_{\mathscr{g}}\right\|_{L^{\infty}(\Omega)} \leq C_{2}\left|\log h_{\mathscr{g}}\right|^{2} \mathscr{\mathscr { C }}_{\mathscr{g}} \quad \forall h_{\mathscr{g}} \leq h^{*},
$$

provided the nondegeneracy condition $\left\|u-u_{\mathscr{G}}\right\|_{L^{\infty}(\Omega)} \geq C h_{\mathscr{G}}^{2}$ holds and $f, \nabla \mathbf{g}$ possess a very weak modulus of continuity within each triangle. We also illustrate the important fact that no term in (1.2) can in general be removed. The upper bound in (1.3) is global, and relies on a novel asymptotic a priori estimate for second derivatives of regularized Green's functions, which is derived in §3. Constant $C_{2}$ is independent of the pole location. The lower bound, which rules out the risk of overestimation, is local, instead, in that a generic element indicator is shown to be bounded above by the pointwise error in the given and certain adjacent triangles. Therefore (1.2) can be used as a basis for an efficient mesh refinement strategy, because excessive overrefinement is very unlikely [19]. We continue in $\S 5$ with the case of point singularities: for $f$ globally continuous and $\mathbf{g}=0$, we prove that $\mathscr{E}_{g}$ can be substituted by

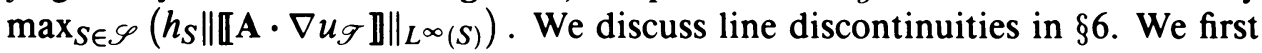


show that $\max _{S \in \mathcal{S}}\left(h_{S}\left\|\llbracket \mathbf{A} \cdot \nabla u_{\mathscr{g}}+\mathbf{g} \rrbracket\right\|_{L^{\infty}(S)}\right)$ is equivalent to $\left\|e_{\mathscr{g}}\right\|_{L^{\infty}(\Omega)}$ for singularities aligned with $\mathscr{T}$. We then study line singularities that may lie within elements, derive an upper bound, and partially examine the issue of overestimation. We finally conclude in $\S 7$ with a thorough discussion of three equivalent error estimators. We demonstrate, for $\mathbf{A}=\mathbf{I}$ and $\mathbf{g}=0$, that the estimators in $[6,24,25]$, when properly interpreted, satisfy a relation similar to (1.3) under the same nondegeneracy and regularity assumptions; no pointwise saturation assumption is needed for [6]. We in fact show their equivalence with $\max _{S \in \mathscr{S}}\left(h_{S}\left|\llbracket \nabla u_{\mathscr{G}} \rrbracket_{S}\right|\right)$, which in turn asserts that all those local estimators extract the same relevant information from $u_{g}$.

We conclude this introduction with a brief discussion of existing literature on pointwise a posteriori error estimation. The estimator of $[10,13]$, developed for $\mathbf{A}=\mathbf{I}$ and $\mathbf{g}=0$, hinges upon a seemingly different idea from those in [2, $3,6,24,25]$. It is based on formally replacing second derivatives of $u$, in the usual a priori error estimates, by discrete second derivatives of $u_{\mathscr{G}}: D_{S}^{2} u_{\mathscr{G}}:=$ $\left|\llbracket \nabla u_{\mathscr{T}} \rrbracket_{S}\right| / h_{S}$. In determining the jumps of $\nabla u_{\mathscr{T}}$, however, the underlying elements are not adjacent but rather sufficiently far apart, at least for theoretical purposes, whereas in practice those jumps are computed across element sides $S$. This severe restriction was subsequently removed in [14], for the energy norm, upon using the residual equation rather than the above approach. Similar results in the maximum norm were announced in the conference report [12] for $\mathbf{A}=\mathbf{I}$ and $\mathbf{g}=0$. Precise assumptions on $\Omega$, indicating whether or not cracks are allowed, along with a substitute for our crucial a priori estimate of $\S 3$ for the Green's function are however missing in [12]. The volumetric residual in (1.2) is claimed to be of higher order than that involving $\llbracket \nabla u_{\mathscr{g}} \rrbracket$, provided $f \in W^{2, \infty}(\Omega)[12]$, which in turn resembles our weaker statement of Theorem 5.1. Since no a posteriori lower bound is discussed in [12, 14], efficiency is assessed via a priori error analysis. This entails convexity of $\Omega$ and mildly graded meshes with mesh density function $h(\mathbf{x})$ satisfying $|\nabla h(\mathbf{x})| \ll 1$ for all $\mathbf{x} \in \Omega$ [11]. These conditions are rarely met in practice.

\section{SetTing}

We now state the precise assumptions on the data and introduce several discrete spaces and local operators, along with the notation to be used throughout the paper. We assume that $\Omega$ is a bounded polygon in $\mathbf{R}^{2}$ without restrictions on the size of the internal angles, that can even be $2 \pi$, and

$$
\begin{gathered}
\mathbf{A}=\left(a_{i j}(\mathbf{x})\right) \text { is positive definite, } a_{i j} \in W^{1, \infty}(\Omega) ; \\
f \in L^{\infty}(\Omega), \quad \mathbf{g} \in\left[B V(\Omega) \cap L^{\infty}(\Omega)\right]^{2} .
\end{gathered}
$$

Additional regularity on $\mathbf{A}, f$, and $\mathbf{g}$ will be imposed later on. A typical $\mathbf{g}$ will exhibit a jump discontinuity across a curve, and will be smooth elsewhere. We will extensively use the notation $\operatorname{osc}_{K} \phi$ for the oscillation of $\phi$ in $K$ and

$$
\langle\phi, \psi\rangle_{K}=\int_{K} \phi \psi, \quad\langle\langle\phi, \mathbf{q}\rangle\rangle_{L}=\int_{L} \phi \mathbf{q} \cdot \mathbf{n}_{L},
$$

where $K$ is a generic subset of $\Omega$ and $L$ is a Lipschitz curve in $\Omega$ with a unit normal vector $\mathbf{n}_{L} ;\langle\cdot, \cdot\rangle$ will stand for the integral over the entire $\Omega$. No ambiguity will arise because of the orientation of $\mathbf{n}_{L}$. We will also indicate 
with $a(\cdot, \cdot)$ the bilinear form

$$
a(\phi, \psi)=\langle\mathbf{A} \cdot \nabla \phi, \nabla \psi\rangle \quad \forall \phi, \psi \in \mathscr{H}:=H_{0}^{1}(\Omega) .
$$

Let $\mathscr{T}$ be a regular partition of $\Omega$ into triangles $T$ with size $h_{T}$, and set $h_{\mathscr{G}}=\max _{T \in \mathscr{G}} h_{T}$ and $\rho_{\mathscr{G}}=\min _{T \in \mathcal{G}} h_{T}\left[8\right.$, p. 124]; $\mathbf{x}_{T}$ denotes the barycenter of $T$. We assume the existence of $\gamma \geq 1$ independent of $\mathscr{T}$ such that

$$
\rho_{\mathscr{T}} \geq C h_{\mathscr{T}}^{\gamma}
$$

and observe that $(2.5)$ is valid in all practical situations. The mesh $\mathscr{T}$ may be highly graded but unstructured: triangles at comparable distance to a singularity are not necessarily of comparable size, as in [10,13,23]. Let $\mathscr{S}$ denote the set of internal interelement boundaries $S$ (or sides), and let $\mathbf{x}_{S}$ indicate the midpoint of $S$ and $h_{S}$ its length. Let $\mathscr{N}:=\left\{\mathbf{x}_{i}\right\}$ be the set of internal nodes of $\mathscr{T}$, and set $\Xi_{i}:=\bigcup\left\{T \in \mathscr{T}: \mathbf{x}_{i} \in T\right\}, \Lambda_{i}:=\bigcup\left\{S \in \mathscr{S}: \mathbf{x}_{i} \in S\right\}$.

Let $\mathscr{P}_{k}(K)$ be the space of polynomials of degree $\leq k$ restricted to $K \subset \Omega$. Let $\mathscr{V}_{\mathscr{g}}^{k} \subset L^{\infty}(\Omega)$ denote the subspace of piecewise discontinuous polynomials of degree $\leq k$, that is $\left.\mathscr{V}_{\mathscr{g}}^{k}\right|_{T}=\mathscr{P}_{k}(T)$, and set $\mathscr{H}_{\mathscr{T}}:=\mathscr{V}_{\mathscr{T}}^{1} \cap \mathscr{H}$. Global continuity is then enforced in $\mathscr{H}_{\mathscr{g}}$. The continuous and discrete solutions, $u$ and $u_{\mathscr{g}}$ respectively, satisfy

$$
\begin{array}{rlr}
u \in \mathscr{H}: & a(u, \phi)=\langle f, \phi\rangle-\langle\mathbf{g}, \nabla \phi\rangle & \forall \phi \in \mathscr{H}, \\
u_{\mathscr{G}} \in \mathscr{H}_{\mathscr{G}}: & a\left(u_{\mathscr{G}}, \varphi\right)=\langle f, \varphi\rangle-\langle\mathbf{g}, \nabla \varphi\rangle & \forall \varphi \in \mathscr{H}_{\mathscr{g}} .
\end{array}
$$

In view of (2.1) and (2.2), $u$ is at least Hölder continuous in $\bar{\Omega}[16,18]$. Given a side $S \in \mathscr{S}, \llbracket \mathbf{q} \rrbracket s^{\cdot \mathbf{n}_{S}}$ denotes the jump of the normal component of $\mathbf{q}$ across $S$, computed in the direction given by $\mathbf{n}_{S}$. With this convention, $\llbracket \mathbf{q} \rrbracket_{S} \cdot \mathbf{n}_{S}$ is independent of the orientation of $\mathbf{n}_{S}$, and it will always be abbreviated as $\llbracket q \rrbracket_{S}$. An elementwise integration by parts shows that $e_{\mathscr{g}}=u-u_{\mathscr{g}}$ verifies the following error or residual equation for all $\phi \in \mathscr{H}$ and $\varphi \in \mathscr{H}_{\mathscr{g}}$ :

$$
\begin{gathered}
a\left(e_{\mathscr{T}}, \phi\right)=\sum_{T \in \mathscr{T}}(\langle f \\
\left.\left.+\operatorname{div} \mathbf{A} \cdot \nabla u_{\mathscr{T}}, \phi-\varphi\right\rangle_{T}-\langle\mathbf{g}, \nabla(\phi-\varphi)\rangle_{T}\right) \\
+\sum_{S \in \mathscr{S}}\left\langle\left\langle\llbracket \mathbf{A} \cdot \nabla u_{\mathscr{T}} \rrbracket_{S}, \phi-\varphi\right\rangle_{S} .\right.
\end{gathered}
$$

Let $P_{\mathscr{T}}: L^{\infty}(\Omega) \rightarrow \mathscr{V}_{\mathscr{T}}^{0}$ be the $L^{2}$-projection operator, which is defined by

$$
\left.P_{\mathscr{G}} \psi\right|_{T} \in \mathscr{P}_{0}(T): \quad\left\langle P_{\mathscr{T}} \psi-\psi, v\right\rangle_{T}=0 \quad \forall v \in \mathscr{P}_{0}(T), T \in \mathscr{T} .
$$

Since $P_{\mathscr{G}}$ is local, standard interpolation theory yields [8]

$$
\left\|P_{\mathscr{T}} \psi-\psi\right\|_{L^{\infty}(T)} \leq \sigma_{\psi}\left(h_{T}\right) \quad \forall T \in \mathscr{T},
$$

where $\sigma_{\psi}$ stands for the modulus of continuity of $\psi$ within each $T$. We also designate with $I_{\mathscr{G}}$ the usual Lagrange interpolation operator on $\mathscr{H}_{\mathscr{G}}$, which is known to satisfy $(2.9)$ as well [8].

Set $\mathscr{W}:=\left[B V(\Omega) \cap L^{\infty}(\Omega)\right]^{2}$ and $\mathscr{W}_{\mathscr{G}}:=\left[\mathscr{V}_{\mathscr{g}}^{1}\right]^{2}$. Let $\Pi_{\mathscr{G}}: \mathscr{W} \rightarrow \mathscr{W}_{\mathscr{G}}$ denote the local projection operator introduced in [7], which, for each $\mathbf{q} \in \mathscr{W}$ and $T \in \mathscr{T}$, is defined by

$$
\left.\Pi_{\mathscr{G}} \mathbf{q}\right|_{T} \in\left[\mathscr{P}_{1}(T)\right]^{2}: \quad\left\langle\left\langle\Pi_{\mathscr{g}} \mathbf{q}-\mathbf{q}, \chi\right\rangle_{S}=0 \quad \forall \chi \in \mathscr{P}_{1}(S),\right.
$$

and for all sides $S \subset \partial T$. Note that $\mathbf{q}$ may be discontinuous in $T$ but its trace is still well defined [17], and that $\prod_{\mathscr{F}} \mathbf{q}$ may exhibit jump discontinuities 
across interelement boundaries. The following well-known local interpolation estimate will be used later [7, 15]:

$$
\left\|\mathbf{q}-\Pi_{\mathscr{g}} \mathbf{q}\right\|_{L^{\infty}(T)}+h_{T}\left\|\operatorname{div}\left(\mathbf{q}-\Pi_{g} \mathbf{q}\right)\right\|_{L^{\infty}(T)} \leq C h_{T} \sigma_{\nabla \mathbf{q}}\left(h_{T}\right) \quad \forall T \in \mathscr{T},
$$

where $\sigma_{\nabla \mathbf{q}}$ stands for the modulus of continuity of $\nabla \mathbf{q}$ within each $T$.

As usual, $C>0$ will denote a generic constant that may vary at the various occurrences, but will always be independent of $u$ and $\mathscr{T}$. The symbol $\approx$ will be used to indicate equivalence, again with lower and upper constants independent of $u$ and $\mathscr{T}$.

\section{Regularized GREeN'S FUNCTIONS}

The purpose of this section is to prove an asymptotic $W^{2, p}$-estimate for regularized Green's functions for general polygonal domains. The a priori bound is uniform with respect to the size of the internal angles of $\Omega$ as $p \downarrow 1$, and is thus valid irrespective of convexity. It is also independent of the pole location.

Let $\delta \in C_{0}^{\infty}(\Omega)$ be a regularization of the Dirac mass satisfying

$$
\begin{gathered}
\operatorname{supp} \delta \subset B:=\left\{\mathbf{x} \in \Omega:\left|\mathbf{x}-\mathbf{x}_{0}\right|<\rho_{0} / 2\right\}, \\
\int_{\Omega} \delta=1, \quad 0 \leq \delta \leq C \rho_{0}^{-2},
\end{gathered}
$$

where $\rho_{0}:=h_{\mathscr{g}}^{\beta}$ and $\mathbf{x}_{0} \in \Omega, \beta>1$ are to be chosen in $\S 4$. Such a function clearly satisfies

$$
\|\delta\|_{L^{p(\Omega)}} \leq C \rho_{0}^{2(1-p) / p} \quad \text { for } p \downarrow 1 .
$$

The corresponding regularized Green's function is defined by

$$
G \in \mathscr{H}: \quad a(G, \phi)=\langle\delta, \phi\rangle \quad \forall \phi \in \mathscr{H} .
$$

The following a priori estimate proved in [18, pp. 206, 233, 266; 9] will be very useful in the sequel:

$$
\|G\|_{W^{2, p_{0}(\Omega)}} \leq C\left(p_{0}, \Omega\right)\|\delta\|_{L^{p_{0}}(\Omega)},
$$

where $1<p_{0}<4 / 3$ is fixed. Note that the restriction $p_{0}<4 / 3$ accounts for the most singular case of slit domains, thereby showing the validity of (3.5) for all bounded polygons. We intend to trace the dependence of $C(p, \Omega)$ on $p$ as $p \downarrow 1$. A by-product of Calderón-Zygmund theory for smooth domains reads

$$
\left\|D^{2} G\right\|_{L^{p}(\Omega)} \leq \frac{C}{p-1}\|\delta\|_{L^{p}(\Omega)} .
$$

It seems, however, that such an estimate is not available in the literature for polygonal domains with reentrant corners. Note that coupling (3.3) and (3.6) results in

$$
\left\|D^{2} G\right\|_{L^{p(\Omega)}} \leq C \frac{\rho_{0}^{-2(p-1)}}{p-1} \quad \text { as } p \downarrow 1 .
$$

We now derive the following slightly weaker result for general polygonal domains.

Theorem 3.1. There holds $\left\|D^{2} G\right\|_{L^{p(\Omega)}} \leq C \frac{\rho_{0}^{-4(p-1)}}{(p-1)^{2}}$ as $p \downarrow 1$.

This estimate is crucial in that it leads to a quasi-optimal $W^{2,1}$-estimate. In fact, on choosing $p=1+\left|\log \rho_{0}\right|^{-1}$ and using $\rho_{0}=h_{\mathscr{G}}^{\beta}$, we get

$$
\left\|D^{2} G\right\|_{L^{\prime}(\Omega)} \leq C\left|\log h_{g}\right|^{2},
$$


Note that the power of the logarithm in (3.7) is one unit higher than expected. This is probably due to the method of proof. Note also that $C$ in (3.7) depends on $\Omega$ and $\beta$ but not on $\mathbf{x}_{0}$. We first demonstrate an auxiliary result.

Lemma 3.1. The following asymptotic bound is valid for $p \downarrow 1$ :

$$
\|G\|_{L^{p /(p-1)}(\Omega)} \leq \frac{C}{(p-1)^{1 / 2}}\|\nabla G\|_{L^{2}(\Omega)} \leq \frac{C}{p-1}\|\delta\|_{L^{p}(\Omega)}, \quad \text { as } p \downarrow 1 .
$$

Proof. We first recall the following 2D Sobolev inequality [16, p. 155, 158]:

$$
\|\phi\|_{L^{q}(\Omega)} \leq C q^{1 / 2}\|\nabla \phi\|_{L^{2}(\Omega)} \quad \forall \phi \in \mathscr{H} .
$$

We then take $\phi=G \in \mathscr{H}$ in (3.4) and make use of Hölder's inequality in conjunction with (3.8) for $q=p /(p-1)$ to deduce

$$
\|\nabla G\|_{L^{2}(\Omega)}^{2} \leq\|\delta\|_{L^{p}(\Omega)}\|G\|_{L^{p /(p-1)}(\Omega)} \leq \frac{C}{(p-1)^{1 / 2}}\|\delta\|_{L^{p}(\Omega)}\|\nabla G\|_{L^{2}(\Omega)} .
$$

This, and a further application of (3.8), concludes the proof.

Proof of Theorem 3.1. Let $d_{j}:=2^{j} \rho_{0}$ for $j \in \mathbf{N}\left(d_{-1}:=0\right)$ and consider the following diadic decomposition of $\Omega$ :

$A_{j}:=\left\{\mathbf{x} \in \Omega: d_{j-1} \leq\left|\mathbf{x}-\mathbf{x}_{0}\right|<d_{j}\right\}, \quad B_{j}:=\left\{\mathbf{x} \in \Omega: d_{j-1} / 2 \leq\left|\mathbf{x}-\mathbf{x}_{0}\right|<2 d_{j}\right\}$. Let $\eta_{j} \in C_{0}^{\infty}\left(B_{j}\right)$ be a cutoff function such that $\eta_{j}=1$ in $A_{j}$ and $\left|D^{k} \eta_{j}\right| \leq$ $C d_{j}^{-k}$. Then, since

$$
\left\|D^{2} G\right\|_{L^{p}(\Omega)}^{p}=\sum_{j}\left\|D^{2} G\right\|_{L^{p}\left(A_{j}\right)}^{p} \leq \sum_{j}\left\|D^{2}\left(G \eta_{j}\right)\right\|_{L^{p}\left(B_{j}\right)}^{p},
$$

we proceed to estimate each term on the right-hand side separately. On using Hölder's inequality, in conjunction with (3.5) for $\eta_{j} G$ and $\mathbf{A} \in\left[W^{1, \infty}(\Omega)\right]^{4}$, we get

$$
\begin{aligned}
& \left\|D^{2}\left(\eta_{j} G\right)\right\|_{L^{p}\left(B_{j}\right)} \leq\left|B_{j}\right|^{\frac{p_{0}-p}{p_{0} p}}\left\|D^{2}\left(\eta_{j} G\right)\right\|_{L^{p_{0}}(\Omega)} \leq C d_{j}^{2 \frac{p_{0}-p}{p_{0} p}}\left\|\operatorname{div}\left(\mathbf{A} \cdot \nabla\left(\eta_{j} G\right)\right)\right\|_{L^{p_{0}}(\Omega)} \\
& \leq C d_{j}^{2 \frac{p_{0}-p}{p_{0} p}}\left(\left\|\eta_{j} \delta\right\|_{L^{p_{0}\left(B_{j}\right)}}+\left\|D \eta_{j} D G\right\|_{L^{p_{0}}\left(B_{j}\right)}+\left\|G D^{2} \eta_{j}\right\|_{L^{p_{0}\left(B_{j}\right)}}\right) \\
& =: I_{j}+I I_{j}+I I I_{j} \text {. }
\end{aligned}
$$

In view of (3.1), $I_{j}=0$ for all $j \geq 1$. In addition, (3.3) for $p_{0}$ yields $\left\|\eta_{0} \delta\right\|_{L^{p_{0}\left(B_{0}\right)}} \leq C \rho_{0}^{2 \frac{1-p_{0}}{p_{0}}}$ and thus

$$
I_{0} \leq C \rho_{0}^{2 \frac{1-p}{p}} .
$$

For the remaining two terms we apply Hölder's inequality, together with $\left|D^{k} \eta_{j}\right|$ $\leq C d_{j}^{-k}$ to arrive at

$$
\begin{aligned}
I I_{j} & \leq C d_{j}^{2 \frac{1-p}{p}}\|D G\|_{L^{2}\left(B_{j}\right)}, \\
I I I_{j} & \leq C d_{j}^{4 \frac{1-p}{p}}\|G\|_{L^{p /(p-1)\left(B_{j}\right)}} .
\end{aligned}
$$

Hence, invoking the finite overlapping property of $\left\{B_{j}\right\}$, Hölder's inequality implies

$$
\sum_{j}\left(I I_{j}^{p}+I I I_{j}^{p}\right) \leq C\|D G\|_{L^{2}(\Omega)}^{p}\left(\sum_{j} d_{j}^{4 \frac{1-p}{2-p}}\right)^{\frac{2-p}{2}}+C\|G\|_{L^{p /(p-1)}(\Omega)}\left(\sum_{j} d_{j}^{4 \frac{1-p}{2-p}}\right)^{2-p} .
$$


Since

$$
\sum_{j} d_{j}^{4 \frac{1-p}{2-p}} \leq \sum_{j} d_{j}^{4(1-p)}=\rho_{0}^{4(1-p)} \sum_{j}\left(16^{1-p}\right)^{j} \leq \frac{\rho_{0}^{4(1-p)}}{1-16^{1-p}} \leq C \frac{\rho_{0}^{4(1-p)}}{p-1}
$$

as $p \downarrow 1$, the asserted estimate is a trivial consequence of (3.3) and Lemma 3.1 .

\section{A POSTERIORI ERROR ANALYSIS}

In this section we prove that a pointwise estimator slightly simpler than that in $\S 1$ is equivalent to $\left\|e_{\mathscr{G}}\right\|_{L^{\infty}(\Omega)}$, provided $f$ and $\mathbf{g}$ are somewhat smooth within each element. To do so, we first examine an estimator applicable even for discontinuous $f$ and $\mathbf{g}$, and show the optimality of our results.

Let $\mathbf{x}_{0} \in \Omega$ satisfy $\left|e_{\mathscr{g}}\left(\mathbf{x}_{0}\right)\right|=\left\|e_{\mathscr{g}}\right\|_{L^{\infty}(\Omega)}$, and let $\delta \geq 0$ denote the regularized Dirac mass of $\S 3$. Our first goal is to prove

$$
\left\|e_{\mathscr{G}}\right\|_{L^{\infty}(\Omega)} \leq C\left|\left\langle e_{\mathscr{G}}, \delta\right\rangle\right|,
$$

for all $h_{\mathscr{G}} \leq h^{*}$ sufficiently small. Given $B$ as in (3.1), let $B_{\mathscr{G}}$ denote the enlarged set

$$
B_{\mathscr{T}}:=\bigcup\{T \in \mathscr{T}: T \cap B \neq \varnothing\} .
$$

Since $\mathscr{T}$ is regular, all triangles of $B_{\mathscr{T}}$ possess comparable size, say $h_{0}$. Select now $\rho_{0}=h_{0}^{\beta}$ in (3.1) with $\beta>2$ to be determined, and let $\mathbf{x}_{1} \in B$ satisfy $\left\langle e_{\mathscr{T}}, \delta\right\rangle=e_{\mathscr{g}}\left(\mathbf{x}_{1}\right)$. We then resort to the Hölder continuity of $u$, say with exponent $0<\alpha \leq 1[9,16,18]$, to deduce

$$
\begin{aligned}
\mid e_{\mathscr{G}}\left(\mathbf{x}_{0}\right) & -e_{\mathscr{G}}\left(\mathbf{x}_{1}\right) \mid \\
& \leq\left|u\left(\mathbf{x}_{0}\right)-u\left(\mathbf{x}_{1}\right)\right|+\left|I_{\mathscr{T}} u\left(\mathbf{x}_{0}\right)-I_{\mathscr{T}} u\left(\mathbf{x}_{1}\right)\right|+\left|I_{\mathscr{G}} e_{\mathscr{T}}\left(\mathbf{x}_{0}\right)-I_{\mathscr{T}} e_{\mathscr{G}}\left(\mathbf{x}_{1}\right)\right| \\
& \leq C \rho_{0}^{\alpha}+C \rho_{0}\left(\left\|\nabla I_{\mathscr{G}} u\right\|_{L^{\infty}(B)}+\left\|\nabla I_{\mathscr{G}} e_{\mathscr{G}}\right\|_{L^{\infty}(B)}\right) \\
& \leq C \rho_{0}^{\alpha}+C \frac{\rho_{0}}{h_{0}}\left(\left\|I_{\mathscr{T}} u-I_{\mathscr{T}} u\left(\mathbf{x}_{0}\right)\right\|_{L^{\infty}\left(B_{\mathcal{G}}\right)}+\left\|I_{\mathscr{G}} e_{\mathscr{G}}\right\|_{L^{\infty}\left(B_{\mathcal{G}}\right)}\right) \\
& \leq C h_{0}^{\beta \alpha}+C h_{0}^{\beta-1}\left\|e_{\mathscr{G}}\right\|_{L^{\infty}(\Omega)},
\end{aligned}
$$

because the oscillation of $u$ is an upper bound for that of $I_{\mathscr{G}} u,\left\|I_{\mathscr{G}} e_{\mathscr{G}}\right\|_{L^{\infty}(T)} \leq$ $\left\|e_{\mathscr{g}}\right\|_{L^{\infty}(T)}$, and $\beta \alpha \leq \beta+\alpha-1$. Hence,

$$
\frac{1}{2}\left\|e_{\mathscr{T}}\right\|_{L^{\infty}(\Omega)} \leq\left(1-C h_{0}^{\beta-1}\right)\left\|e_{\mathscr{T}}\right\|_{L^{\infty}(\Omega)} \leq C h_{0}^{\beta \alpha}+\left|\left\langle e_{\mathscr{T}}, \delta\right\rangle\right|,
$$

for $h_{0} \leq h_{\mathscr{T}} \leq h^{*}$ sufficiently small. Unless $u$ is globally linear (a trivial case!), we can always assume the existence of an element $\hat{T} \in \mathscr{T}$ satisfying $\left\|u-I_{\mathscr{T}} u\right\|_{L^{\infty}(\hat{T})} \geq C h_{\hat{T}}^{2}$ : a sufficient condition is $\pm u_{x_{i} x_{i}}(x) \geq C>0$ for all $x \in \hat{T}$. With the aid of $(2.5)$, we infer that

$$
C h_{\mathscr{G}}^{2 \gamma} \leq C h_{\hat{T}}^{2} \leq C\left\|u-I_{\mathscr{T}} u\right\|_{L^{\infty}(\hat{T})} \leq\left\|e_{\mathscr{T}}\right\|_{L^{\infty}(\Omega)} .
$$

On choosing $\beta>2 \gamma / \alpha$, the first term on the right-hand side of (4.3) can be hidden into the left, thereby leading to (4.1). We stress that both $C$ and $h^{*}$ in (4.1) depend on $\mathbf{A}, f, \mathbf{g}$, and $\Omega$ but not on $u$ nor on $\mathscr{T}$, except for (2.5). 
The error equation (2.7) can be written equivalently as follows:

$$
\begin{aligned}
& a\left(e_{\mathscr{G}}, \phi\right)=\sum_{T \in \mathscr{T}}\left\langle f+\operatorname{div} \mathbf{A} \cdot \nabla u_{\mathscr{G}}+\operatorname{div} \Pi_{\mathscr{T}} \mathbf{g}, \phi-\varphi\right\rangle_{T} \\
& -\sum_{T \in \mathscr{T}}\left\langle\mathbf{g}-\Pi_{\mathscr{G}} \mathbf{g}, \nabla(\phi-\varphi)\right\rangle_{T} \\
& +\sum_{S \in \mathscr{S}}\left\langle\left\langle\llbracket \mathbf{A} \cdot \nabla u_{\mathscr{T}}+\Pi_{\mathscr{T}} \mathbf{g} \rrbracket_{S}, \phi-\varphi\right\rangle_{S},\right.
\end{aligned}
$$

for all $\phi \in \mathscr{H}$ and $\varphi \in \mathscr{H}_{\mathscr{g}}$. This suggests considering the pointwise indicator

$$
\begin{aligned}
E_{T}^{1}:= & h_{T}^{2}\left\|f+\operatorname{div} \mathbf{A} \cdot \nabla u_{\mathscr{G}}+\operatorname{div} \Pi_{\mathscr{T}} \mathbf{g}\right\|_{L^{\infty}(T)} \\
& +h_{T}\left\|\mathbf{g}-\Pi_{\mathscr{T}} \mathbf{g}\right\|_{L^{\infty}(T)}+h_{T}\left\|\llbracket \mathbf{A} \cdot \nabla u_{\mathscr{G}}+\Pi_{\mathscr{G}} \mathbf{g} \rrbracket\right\|_{L^{\infty}(\partial T)},
\end{aligned}
$$

and corresponding pointwise estimator $\mathscr{E}_{\mathscr{G}}^{1}:=\max _{T \in \mathscr{T}} E_{T}^{1}$.

Theorem 4.1. There exist constants $C_{1}, C_{2}, C_{3}, C_{4}, h^{*}>0$ independent of $u$ and $\mathscr{I}$, such that for all $h_{\mathscr{G}} \leq h^{*}$ the following estimates are valid:

$$
\begin{aligned}
& C_{1}\left|\log h_{\mathscr{G}}\right|^{2} \mathscr{E}_{\mathscr{g}}^{1} \geq\left\|e_{\mathscr{g}}\right\|_{L^{\infty}(\Omega)} \\
& \geq C_{2} \mathscr{E}_{\mathscr{G}}^{1}-C_{3} \max _{T \in \mathscr{T}}\left(h_{T}^{2}\left\|f-P_{\mathscr{G}} f\right\|_{L^{\infty}(T)}\right. \\
& \left.+h_{T}^{2}\left\|\operatorname{div}\left(\mathbf{A}-I_{\mathscr{G}} \mathbf{A}\right) \cdot \nabla u_{\mathscr{G}}\right\|_{L^{\infty}(T)}+h_{T}\left\|\mathbf{g}-\Pi_{\mathscr{G}} \mathbf{g}\right\|_{L^{\infty}(T)}\right) \\
& -C_{4} \max _{S \in \mathscr{S}}\left(h_{S}\left\|\left(\mathbf{A}-I_{\mathscr{S}} \mathbf{A}\right) \cdot \llbracket \nabla u_{\mathscr{S}} \mathbb{1}_{S}\right\|_{L^{\infty}(S)}\right) \text {. }
\end{aligned}
$$

Note that the logarithmic factor can be considered bounded for practical purposes. The following 1D examples illustrate the crucial fact that no term in (4.5) can be dropped. Let $\Omega:=(-1,1)$ and $\mathscr{T}$ be a uniform mesh with an even number of subintervals of size $h$, and let $\mathbf{A}=1$. The functions $f$, $g=\mathbf{g}$, and $u$ are $2 h$-periodic in the first two examples.

Example 4.1. Let $g=0$ and $f$ be the odd function given by $f(x)=1$ for $0<x<h$. Then $u$ turns out to be odd and given by $u(x)=x(h-x) / 2$ for $0<x<h$, whereas $u_{\mathscr{g}}=0$. Therefore $\left\|e_{\mathscr{g}}\right\|_{L^{\infty}(\Omega)}=\frac{h^{2}}{8}\|f\|_{L^{\infty}(\Omega)}=\frac{1}{8} \mathscr{E}_{\mathscr{g}}$.

Example 4.2. Let $f=0$ and $g$ be the even function defined by $g(x)=1$ for $0<x<h / 2$ and $g(x)=-1$ for $h / 2<x<h$. Now $u$ is odd and reads $u(x)=|x-h / 2|-h / 2$ for $0<x<h$, whereas $\Pi_{g} g$ is continuous and expressed by $\Pi_{\mathscr{g}} g(x)=1-2|x| / h$ within $(-h, h)$. This leads to $u_{\mathscr{G}}=0$, $\left(\Pi_{\mathscr{G}} g\right)^{\prime}= \pm 2 / h, \llbracket \Pi_{\mathscr{G}} g \rrbracket=0$, and $\left\|e_{\mathscr{G}}\right\|_{L^{\infty}(\Omega)}=\frac{h}{2}\left\|g-\Pi_{\mathscr{G}} g\right\|_{L^{\infty}(\Omega)}=\frac{1}{2} \mathscr{E}_{\mathscr{G}}$.

Example 4.3. Let $u(x)=1-|x|, f(x)=0$, and $g(x)=\operatorname{sgn}(x)$. Then $u_{\mathcal{G}}=u, \Pi_{g} g=g$, and $\left\|e_{g}\right\|_{L^{\infty}(\Omega)}=\max _{i}\left|\llbracket u_{\mathscr{g}}^{\prime}+g \rrbracket\left(x_{i}\right)\right|=0$, whereas $\max _{i}\left|\llbracket u_{\mathscr{G}}^{\prime} \rrbracket\left(x_{i}\right)\right|=\left|\llbracket u_{g}^{\prime} \rrbracket(0)\right|=2$.

The following example demonstrates that the factor multiplying $C_{3}$ cannot in general be removed, and consequently that $\mathscr{E}_{\mathscr{g}} \frac{1}{}$ may overestimate the pointwise error.

Example 4.4. Consider $f$ and $g$ as in the first two examples but with period $2 h / N$, where $N \geq 2$ indicates an even integer. The error $\left\|e_{g}\right\|_{L^{\infty}(\Omega)}$ becomes 
$\mathscr{O}\left(h^{2} / N^{2}\right)($ or $\mathscr{O}(h / N))$, whereas $P_{\mathscr{g}} f=0$ (or $\Pi_{\mathscr{g}} g=1$ ) and $\mathscr{\mathscr { E } _ { \mathscr { g } }}=h^{2}$ (or $=h$ ) does not change with $N$.

The proof of (4.6) will be split into two lemmas. We start out by showing the above upper bound. To this end we use a global argument.

Lemma 4.1. There holds $\left\|e_{\mathscr{g}}\right\|_{L^{\infty}(\Omega)} \leq C\left|\log h_{\mathscr{G}}\right|^{2 \mathscr{E} 1}$ for all $h_{\mathscr{G}} \leq h^{*}$.

Proof. Let $\phi=G \in \mathscr{H}$ be the test function in (4.4), where $G$ stands for the regularized Green's function of $\S 3$. Interpolation theory in $L^{1}(\Omega)$ [8], combined with (3.7) and (4.4), yields

$$
\left|\left\langle e_{\mathscr{G}}, \delta\right\rangle\right|=\left|a\left(e_{\mathscr{G}}, G\right)\right| \leq C \mathscr{E}_{\mathscr{G}}^{1}\left\|D^{2} G\right\|_{L^{1}(\Omega)} \leq C\left|\log h_{\mathscr{T}}\right|^{2} \mathscr{E}_{\mathscr{G}}^{1} .
$$

The assertion then follows from (4.1).

The constant $C$ in Lemma 4.1 is rather difficult to calculate because of its relation with the Green's function, but is independent of the location of the pole $\mathbf{x}_{0}$. Despite the moderate size of $C$ [10], its concrete quantification deserves further investigation.

The following proof is in essence a modification of a local argument by Verfürth [24], which carries over regardless of the magnitude of the local error. For any $T_{0} \in \mathscr{T}$, set $h_{0}:=h_{T_{0}}$ and let $T_{0}^{*}$ be the enlarged set

$$
T_{0}^{*}:=\bigcup\left\{T \in \mathscr{T}: T \text { and } T_{0} \text { have a common side }\right\}
$$

Lemma 4.2. The following lower bound holds for all $h_{0} \leq h^{*}$ and $T_{0} \in \mathscr{T}$ :

$$
\begin{aligned}
E_{T_{0} \leq}^{1} \leq & C\left\|e_{\mathscr{G}}\right\|_{L^{\infty}\left(T_{0}^{*}\right)} \\
& +C \max _{T \subset T_{0}^{*}}\left(h_{T}^{2}\left\|f-P_{\mathscr{T}} f\right\|_{L^{\infty}(T)}+h_{T}^{2}\left\|\operatorname{div}\left(\mathbf{A}-I_{\mathscr{T}} \mathbf{A}\right) \cdot \nabla u_{\mathscr{T}}\right\|_{L^{\infty}(T)}\right. \\
& \left.+h_{T}\left\|\mathbf{g}-\Pi_{\mathscr{T}} \mathbf{g}\right\|_{L^{\infty}(T)}\right) \\
& +C \max _{S \subset \partial T_{0}}\left(h_{S}\left\|\left(\mathbf{A}-I_{\mathscr{T}} \mathbf{A}\right) \cdot \llbracket \nabla u_{\mathscr{T}} \rrbracket_{S}\right\|_{L^{\infty}(S)}\right) .
\end{aligned}
$$

Proof. In order to localize the analysis, we deal with a test function $v \in$ $W^{1, \infty}(\Omega)$ whose support is contained in $T_{0}^{*}$. The explicit construction of $v$ proceeds as follows. Set

$$
\begin{aligned}
& q_{S}:=\llbracket I_{\mathscr{T}} \mathbf{A} \cdot \nabla u_{\mathscr{T}}+\Pi_{\mathscr{T}} \mathbf{g} \rrbracket_{S} \cdot \mathbf{n}_{S}, \\
& F_{T}:=\left.P_{\mathscr{G}} f\right|_{T}+\left.\operatorname{div}\left(I_{\mathscr{G}} \mathbf{A}\right) \cdot \nabla u_{\mathscr{G}}\right|_{T}+\left.\operatorname{div} \Pi_{\mathscr{G}} \mathbf{g}\right|_{T} .
\end{aligned}
$$

Note that $q_{S}$ is linear in $S \in \mathscr{S}$ whereas $F_{T}$ is constant in $T \in \mathscr{T}$. We seek a piecewise polynomial function $v$ satisfying $v=0$ on $\partial T_{0}^{*}$ and

$$
\begin{array}{ll}
\left\langle\left\langle q_{S}, v\right\rangle_{S}=h_{S}\left\|q_{S}\right\|_{L^{\infty}(S)}\right. & \forall S \subset \partial T_{0}, \\
\left\langle F_{T}, v\right\rangle_{T}=h_{T}^{2}\left\|F_{T}\right\|_{L^{\infty}(T)} & \forall T \subset T_{0}^{*} .
\end{array}
$$


Let $b_{T} \in \mathscr{P}_{3}(T)$ be the canonical bubble function of $T$, i.e., the product of the barycentric coordinates of $T$. Let $\varphi_{S}$ be the canonical basis function of $\mathscr{P}_{2}(T)$ that vanishes at all nodes of $\mathscr{T}$ and midpoints of $\mathscr{S}$ but $\mathbf{x}_{S} \in S \subset$ $\partial T$, at which $\varphi_{S}\left(\mathbf{x}_{S}\right)=1$. For each $S \subset \partial T_{0}$, we still denote by $q_{S}$ the linear extension of $q_{S}$ to $T_{0}^{*}$ that vanishes at the opposite vertices. Consider $v \in W^{1, \infty}\left(T_{0}^{*}\right)$ of the form

$$
v=\sum_{T \subset T_{0}^{*}} \alpha_{T} b_{T}+\sum_{S \subset \partial T_{0}} \beta_{S} q_{S} \varphi_{S}
$$

where $\left\{\alpha_{T}\right\}$ and $\left\{\beta_{S}\right\}$ are determined as follows. With such a $v,(4.8)$ reads

$$
\beta_{S}\left\langle\left\langle q_{S}^{2}, \varphi_{S}\right\rangle\right\rangle_{S}=h_{S}\left\|q_{S}\right\|_{L^{\infty}(S)},
$$

which yields a unique $\beta_{S}$. Moreover, since $\left\langle\left\langle q_{S}^{2}, \varphi_{S}\right\rangle_{S} \approx h_{S}\left\|q_{S}\right\|_{L^{\infty}(S)}^{2}\right.$ as a consequence of $q_{S}$ being linear and $\varphi_{S}>0$ and quadratic, we see that

$$
\left|\beta_{S}\right|\left\|q_{S}\right\|_{L^{\infty}(S)} \leq C \text {. }
$$

Since $F_{T} \in \mathscr{P}_{0}(T)$, (4.9) becomes

$$
\alpha_{T} F_{T}\left\langle b_{T}, 1\right\rangle_{T}=h_{T}^{2}\left\|F_{T}\right\|_{L^{\infty}(T)}-F_{T} \sum_{S \subset \partial T_{0}} \beta_{S}\left\langle q_{S}, \varphi_{S}\right\rangle_{T},
$$

which in turn defines $\alpha_{T}$ uniquely. Since $b_{T}>0$ is cubic, we have $\left\langle b_{T}, 1\right\rangle_{T} \approx$ $h_{T}^{2}$, and thus

$$
\left|\alpha_{T}\right| \leq C\left(1+\sum_{S \subset \partial T_{0}}\left|\beta_{S}\right|\left\|q_{S}\right\|_{L^{\infty}(T)}\left\|\varphi_{S}\right\|_{L^{\infty}(T)}\right) \leq C .
$$

Extend $v$ by zero outside $T_{0}^{*}$ and use the fact that $\varphi_{S}=b_{T}=0$ outside $T_{0}^{*}$ to conclude that $v \in W_{0}^{1, \infty}(\Omega)$. Invoking local inverse inequalities for $v$, which is piecewise polynomial, and making use of (4.10) and (4.11), leads to the a priori bound

$$
\left\|D^{2} v\right\|_{L^{\prime}(T)} \leq C h_{T}^{-2}\|v\|_{L^{\prime}(T)} \leq C\|v\|_{L^{\infty}(T)} \leq C .
$$

Since $I_{g} v=0$, we can write $v=v-I_{g} v$ and then use interpolation theory, in conjunction with a standard trace inequality, to obtain

$$
\begin{aligned}
\|v\|_{L^{\prime}(T)} & +h_{T}\|\nabla v\|_{L^{\prime}(T)} \\
& +h_{T}\|v\|_{L^{\prime}(\partial T)}+h_{T}^{2}\|\partial v / \partial n\|_{L^{\prime}(\partial T)} \leq C h_{T}^{2}\left\|D^{2} v\right\|_{L^{\prime}(T)} \leq C h_{T}^{2} .
\end{aligned}
$$

With $\phi=v$ in (4.4), and the aid of (4.8), (4.9), (4.12), (2.1), and integration 
by parts, we immediately get

$$
\begin{aligned}
& \sum_{T \subset T_{0}^{*}} h_{T}^{2}\left\|F_{T}\right\|_{L^{\infty}(T)}+\sum_{S \subset \partial T_{0}} h_{S}\left\|q_{S}\right\|_{L^{\infty}(S)} \\
& =\sum_{T \in \mathscr{T}}\left\langle F_{T}, v\right\rangle_{T}+\sum_{S \in \mathscr{S}}\left\langle\left\langle q_{S}, v\right\rangle_{S}\right. \\
& =a\left(e_{g}, v\right) \\
& +\sum_{T \in \mathscr{T}}\left(\left\langle P_{\mathscr{T}} f-f, v\right\rangle_{T}+\left\langle\operatorname{div}\left(I_{\mathscr{G}} \mathbf{A}-\mathbf{A}\right) \cdot \nabla u_{\mathscr{T}}, v\right\rangle_{T}+\left\langle\mathbf{g}-\Pi_{\mathscr{T}} \mathbf{g}, \nabla v\right\rangle_{T}\right) \\
& +\sum_{S \in \mathscr{S}}\left\langle\left\langle\mathbb{I}\left(I_{\mathscr{T}} \mathbf{A}-\mathbf{A}\right) \cdot \nabla u_{\mathscr{T}} \rrbracket_{S}, v\right\rangle_{S}\right. \\
& =-\sum_{T \in \mathscr{T}}\left\langle e_{\mathscr{T}}, \operatorname{div}(\mathbf{A} \cdot \nabla v)\right\rangle_{T}-\sum_{S \in \mathscr{S}}\left\langle\left\langle e_{\mathscr{T}}, \llbracket \mathbf{A} \cdot \nabla v \rrbracket_{S}\right\rangle_{S}\right. \\
& +\sum_{T \in \mathscr{T}}\left(\left\langle P_{\mathscr{G}} f-f, v\right\rangle_{T}+\left\langle\operatorname{div}\left(I_{\mathscr{T}} \mathbf{A}-\mathbf{A}\right) \cdot \nabla u_{\mathscr{G}}, v\right\rangle_{T}+\left\langle\mathbf{g}-\Pi_{\mathscr{G}} \mathbf{g}, \nabla v\right\rangle_{T}\right) \\
& +\sum_{S \in \mathscr{S}}\left\langle\left\langle\mathbb{\amalg}\left(I_{\mathscr{T}} \mathbf{A}-\mathbf{A}\right) \cdot \nabla u_{\mathscr{T}} \rrbracket_{S}, v\right\rangle_{S}\right. \\
& \leq C\left\|e_{g}\right\|_{L^{\infty}\left(T_{0}^{*}\right)} \\
& +C \max _{T \subset T_{0}^{*}}\left(h_{T}^{2}\left\|f-P_{\mathscr{G}} f\right\|_{L^{\infty}(T)}+h_{T}^{2}\left\|\operatorname{div}\left(I_{\mathscr{G}} \mathbf{A}-\mathbf{A}\right) \cdot \nabla u_{\mathscr{G}}\right\|_{L^{\infty}(T)}\right. \\
& \left.+h_{T}\left\|\mathbf{g}-\Pi_{\mathscr{g}} \mathbf{g}\right\|_{L^{\infty}(T)}\right) \\
& +C \max _{S \subset \partial T_{0}}\left(h_{S}\left\|\left(\mathbf{A}-I_{\mathscr{G}} \mathbf{A}\right) \cdot \llbracket \nabla u_{\mathscr{G}} \rrbracket_{S}\right\|_{L^{\infty}(S)}\right) \text {. }
\end{aligned}
$$

Adding and subtracting $f$ and $\operatorname{div} \mathbf{A} \cdot \nabla u_{g}$ to $F_{T}$ in the first term of the previous expression, and $\llbracket \mathbf{A} \cdot \nabla u_{\mathscr{G}} \rrbracket_{S} \cdot \mathbf{n}_{S}$ to $q_{S}$ in the second, one easily obtains the assertion.

Note that without additional regularity assumptions, the above construction may produce a poor lower bound. In fact, $u_{\mathscr{T}}=0$ and $v=0$ for the Example 4.4 because either $P_{\mathscr{g}} f=0$ or $\Pi_{\mathscr{g}} \mathbf{g}=1$, so

$$
E_{T_{0}}^{1} \approx \max _{T \subset T_{0}^{*}}\left(h_{T}^{2}\left\|f-P_{\mathscr{G}} f\right\|_{L^{\infty}(T)}+h_{T}\left\|\mathbf{g}-\Pi_{\mathscr{g}} \mathbf{g}\right\|_{L^{\infty}(T)}\right) .
$$

This obviously gives no lower bound for $\left\|e_{\mathscr{G}}\right\|_{L^{\infty}(\Omega)}$ and raises the question of a possible overestimation, which is again confirmed by Example 4.4.

Our aim now is to show that overestimation cannot occur whenever $f$ and $\nabla \mathbf{g}$ are uniformly continuous and $\mathbf{A} \in W^{2, \infty}$, both elementwise, and the following nondegeneracy assumption is valid:

$$
C h_{\mathscr{g}}^{2} \leq\left\|e_{\mathscr{T}}\right\|_{L^{\infty}(\Omega)} .
$$

It is worth stressing that discontinuities of $f, \mathbf{g}$, and $\operatorname{div} \mathbf{A}$ are still allowed across interelement boundaries, and that (4.14) is quite reasonable in applications: it is sufficient to have $\pm u_{x_{i} x_{i}} \geq C>0$ in a triangle of size $h_{\mathscr{g}}$. In this setup, the simpler local indicator

$$
E_{T}^{2}:=h_{T}^{2}\left|f+\operatorname{div} \mathbf{A} \cdot \nabla u_{\mathcal{G}}+\operatorname{div} \mathbf{g}\right|\left(\mathbf{x}_{T}\right)+h_{T}\left\|\llbracket \mathbf{A} \cdot \nabla u_{\mathcal{G}}+\mathbf{g} \rrbracket\right\|_{L^{\infty}(\partial T)}
$$

makes sense, and gives rise to the pointwise estimator $\mathscr{E}_{\mathscr{g}}^{2}:=\max _{T \in \mathcal{G}} E_{T}^{2}$. 
Theorem 4.2. Let $\mathbf{A} \in\left[W^{2, \infty}(T)\right]^{4}$ for all $T \in \mathscr{T}$. Let both $f$ and $\nabla \mathbf{g}$ be uniformly continuous in each triangle $T \in \mathscr{T}$, and let their moduli of continuity satisfy $\sigma_{f}(t), \sigma_{\nabla \mathbf{g}}(t)=o\left(|\log t|^{-2}\right)$. If (4.14) holds, then there exist $C_{1}, C_{2}, h^{*}>0$ independent of $u$ and $\mathscr{T}$ such that

$$
C_{1} \mathscr{E}_{\mathscr{g}}^{2} \leq\left\|e_{\mathscr{G}}\right\|_{L^{\infty}(\Omega)} \leq C_{2}\left|\log h_{\mathscr{G}}\right|^{2} \mathscr{E}_{\mathscr{G}}^{2} \quad \forall h_{\mathscr{G}} \leq h^{*} .
$$

Proof. Let $T_{0} \in \mathscr{T}$ satisfy $E_{T_{0}}^{1}=\mathscr{\mathscr { C }} \mathscr{g}$ and set $h_{0}:=h_{T_{0}}, \mathbf{x}_{0}:=\mathbf{x}_{T_{0}}$. Since A is globally $W^{1, \infty}$ and locally $W^{2, \infty}$, we deduce that $\left.\mathbf{A}\right|_{S} \in W^{2, \infty}(S)$ for all $S \in \mathscr{S}$. Hence,

$$
\underset{T_{0}}{\operatorname{osc}}|\operatorname{div} \mathbf{A}| \leq C h_{0}, \quad\left\|\mathbf{A}-I_{\mathscr{G}} \mathbf{A}\right\|_{L^{\infty}\left(\partial T_{0}\right)} \leq C h_{0}^{2} .
$$

Lemma 4.1, together with (4.14), (4.17), and

$$
\|\llbracket \chi \rrbracket\|_{L^{\infty}\left(\partial T_{0}\right)} \leq 2\|\chi\|_{L^{\infty}\left(T_{0}^{*}\right)}
$$

for $\chi=\mathbf{g}-\Pi_{g} \mathbf{g}$, leads to

$$
\begin{aligned}
& C h_{0}^{2} \leq C h_{\mathscr{g}}^{2} \leq\left\|e_{\mathscr{T}}\right\|_{L^{\infty}(\Omega)} \leq C\left|\log h_{0}\right|^{2} E_{T_{0}}^{1} \\
& \leq C h_{0}\left|\log h_{0}\right|^{2}\left(h_{0}\left|f+\operatorname{div} \mathbf{A} \cdot \nabla u_{\mathscr{G}}+\operatorname{div} \mathbf{g}\right|\left(\mathbf{x}_{0}\right)+\left\|\llbracket \mathbf{A} \cdot \nabla u_{\mathscr{G}}+\mathbf{g} \rrbracket\right\|_{L^{\infty}\left(\partial T_{0}\right)}\right) \\
& +C h_{0}\left|\log h_{0}\right|^{2}\left(h_{0}\left\|f-f\left(\mathbf{x}_{0}\right)\right\|_{L^{\infty}\left(T_{0}\right)}+\left\|\mathbf{g}-\Pi_{\mathscr{G}} \mathbf{g}\right\|_{L^{\infty}\left(T_{0}^{*}\right)}\right. \\
& \left.+h_{0}\left\|\operatorname{div}\left(\mathbf{g}-\Pi_{g} \mathbf{g}\right)\right\|_{L^{\infty}\left(T_{0}\right)}\right) \\
& +C h_{0}^{3}\left|\log h_{0}\right|^{2}\left\|\nabla u_{\mathscr{G}}\right\|_{L^{\infty}\left(T_{0}\right)} .
\end{aligned}
$$

We then use (2.9) and (2.11) to deduce that all terms in the third line are $o\left(h_{0}^{2}\right)$, and thus asymptotically negligible. Since $u \in C^{\alpha}\left(\overline{T_{0}^{*}}\right)$ for some $0<\alpha \leq 1$ depending solely on $\Omega, f$ and $\mathbf{g}[9,16,18]$, we see that

$$
\begin{aligned}
h_{0}^{3}\left\|\nabla u_{\mathcal{G}}\right\|_{L^{\infty}\left(T_{0}\right)} & \leq C h_{0}^{2} \underset{T_{0}}{\operatorname{osc}} u_{\mathcal{G}} \\
& \leq C h_{0}^{2} \underset{T_{0}}{\operatorname{osc}} e_{\mathscr{T}}+C h_{0}^{2} \underset{T_{0}}{\operatorname{osc}} u \leq C h_{0}^{2}\left\|e_{\mathscr{G}}\right\|_{L^{\infty}(\Omega)}+C h_{0}^{2+\alpha} .
\end{aligned}
$$

We realize that these two terms, multiplied by $\left|\log h_{0}\right|^{2}$, are negligible with respect to $\left\|e_{\mathscr{G}}\right\|_{L^{\infty}(\Omega)}$. The fact that $h_{0} \geq \rho_{\mathscr{G}} \geq C h_{\mathscr{G}}^{\gamma}$ thus yields the upper bound in (4.16).

To prove the lower bound in (4.16), let $T_{0} \in \mathscr{T}$ satisfy $E_{T_{0}}^{2}=\mathscr{E}_{\mathscr{g}}^{2}$. We again argue as above, now using Lemma 4.2 in conjunction with (2.9), (2.11), (4.17), (4.18) with $\chi=\llbracket \nabla u_{\mathscr{G}} \rrbracket$, and (4.19) for $T_{0}^{*}$, to obtain

$$
\begin{aligned}
\mathscr{E}_{\mathscr{G}}^{2}=E_{T_{0}}^{2} & \leq E_{T_{0}}^{1}+h_{0}^{3}\left|\log h_{0}\right|^{2}\left\|\nabla u_{\mathscr{G}}\right\|_{L^{\infty}\left(T_{0}^{*}\right)}+o\left(h_{\mathscr{G}}^{2}\left|\log h_{\mathscr{G}}\right|^{-2}\right) \\
& \leq C\left\|e_{\mathscr{G}}\right\|_{L^{\infty}(\Omega)}+o\left(h_{\mathscr{G}}^{2}\left|\log h_{\mathscr{G}}\right|^{-2}\right) \leq C\left\|e_{\mathscr{G}}\right\|_{L^{\infty}(\Omega)} .
\end{aligned}
$$

The constant $C_{1}$ in (4.16) can be computed explicitly because it involves the solution of local problems. We refer to [1] for an analysis in the energy norm.

Remark 4.1. We would like to stress the local character of the estimate (4.7). In fact, if we knew that

$$
C h_{0}^{2} \leq\left\|e_{\mathscr{G}}\right\|_{L^{\infty}\left(T_{0}\right)},
$$


we could then repeat the argument in Theorem 4.2 and obtain the following local result:

$$
C_{1} E_{T_{0}}^{2} \leq\left\|e_{g}\right\|_{L^{\infty}\left(T_{0}^{*}\right)} .
$$

Therefore, an adaptive strategy for mesh refinement could in principle be based on (4.15) and it would be efficient in the sense of [19], that is, overrefinement would be avoided in view of (4.21). The nondegeneracy assumption (4.20) is guaranteed whenever $\pm u_{x_{i} x_{i}} \geq C>0$ in $T_{0}$, but pathological situations arising from numerical pollution cannot be excluded.

In the next two sections we will investigate the relative importance of the jump residual in (4.15). We will prove in $\S 5$ that the local estimator

$$
\mathscr{E}_{\mathscr{G}}^{3}:=\max _{S \in \mathscr{S}}\left(h_{S}\left\|\llbracket \mathbf{A} \cdot \nabla u_{\mathscr{G}} \rrbracket\right\|_{L^{\infty}(S)}\right) \text {, }
$$

is equivalent to $\left\|e_{\mathscr{g}}\right\|_{L^{\infty}(\Omega)}$ provided $\mathbf{g}=0$. Example 4.1 suggests that such an undertaking would only be possible under global continuity of $f$. If $\mathbf{g} \neq 0$ and exhibits jump discontinuities across element sides only, then Example 4.3 indicates that

$$
\mathscr{E}_{\mathscr{G}}^{4}:=\max _{S \in \mathscr{S}}\left(h_{S}\left\|\llbracket \mathbf{A} \cdot \nabla u_{\mathscr{G}}+\mathbf{g} \rrbracket\right\|_{L^{\infty}(S)}\right)
$$

may be equivalent to $\left\|e_{\mathscr{g}}\right\|_{L^{\infty}(\Omega)}$, which is in fact shown in $\S 6$. The case of discontinuities not aligned with $\mathscr{T}$, along with the possibility of overestimation, will also be studied in $\S 6$.

\section{CASE $\mathbf{g}=0:$ POINT SINGUlarities}

We now assume $\mathbf{g}=0$ and intend to remove the residual term involving $f+\operatorname{div} \mathbf{A} \cdot \nabla u_{g}$ in (4.15). Even though $f$ is bounded, and so $u \in C^{1, \alpha}$ locally for all $0<\alpha<1$ [16], the need for mesh refinements and a posteriori error control may be due to the pollution effect created by corner (or point) singularities $[9,10,18,23]$.

Theorem 5.1. Let $\mathbf{A} \in\left[W^{2, \infty}(\Omega)\right]^{4}$ and let the modulus of continuity of $f$ in the entire $\Omega$ satisfy $\sigma_{f}(t)=o\left(|\log t|^{-2}\right)$. If (4.14) holds, then there exist constants $C_{1}, C_{2}, h^{*}>0$ independent of $u$ and $\mathscr{T}$ such that

$$
C_{1} \mathscr{E}_{\mathscr{G}}^{3} \leq\left\|e_{\mathscr{G}}\right\|_{L^{\infty}(\Omega)} \leq C_{2}\left|\log h_{\mathscr{T}}\right|^{2} \mathscr{E}_{\mathscr{G}}^{3} \quad \forall h_{\mathscr{T}} \leq h^{*} .
$$

Proof. Let $\mathbf{x}_{i} \in \mathcal{N}$ be a generic node and $\varphi_{i}$ be the canonical basis function associated with it. Take $\varphi=\varphi_{i}$ as a test function in (2.6) and integrate by parts to arrive at

$$
\left\langle R_{i}, \varphi_{i}\right\rangle_{\Xi_{i}}+\left\langle\left\langle\llbracket \mathbf{A} \cdot \nabla u_{\mathscr{g}} \rrbracket, \varphi_{i}\right\rangle\right\rangle_{\Lambda_{i}}=\left\langle R_{i}-R, \varphi_{i}\right\rangle_{\Xi_{i}},
$$

where $R:=f+\operatorname{div} \mathbf{A} \cdot \nabla u_{g}$ is the residual and $R_{i}:=R\left(\mathbf{x}_{T_{i}}\right)$ with $T_{i} \subset \Xi_{i}$ fixed. Since $\left\langle R_{i}, \varphi_{i}\right\rangle_{\Xi_{i}}=R_{i} \int_{\Xi_{i}} \varphi_{i}=C h_{i}^{2} R_{i}$ with $h_{i}$ indicating the size of $\Xi_{i}$, (2.1) and (5.2) lead to

$$
\left|R_{i}\right| \leq\left\|R-R_{i}\right\|_{L^{\infty}\left(\Xi_{i}\right)}+C h_{i}^{-1}\left\|\llbracket \mathrm{A} \cdot \nabla u_{\mathscr{g}} \rrbracket\right\|_{L^{\infty}\left(\Lambda_{i}\right)} .
$$

With the aid of $(2.1)$ and the fact that $\llbracket \nabla u_{g} \rrbracket s$ is parallel to $\mathbf{n}_{S}$, we realize that $\left|\mathbf{n}_{S} \cdot \mathbf{A} \cdot \llbracket \nabla u_{\mathscr{G}} \rrbracket_{S}\right| \geq C\left|\llbracket \nabla u_{\mathscr{G}} \rrbracket_{S}\right|$. Since card $\Lambda_{i} \leq C$ is independent of $\mathscr{T}$, we can write

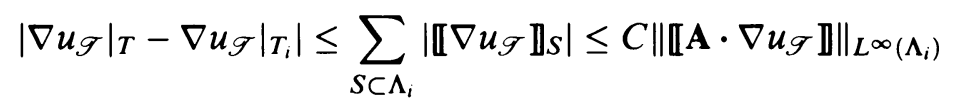


for all $T \subset \Xi_{i}$, and thus

$$
\left\|R-R_{i}\right\|_{L^{\infty}\left(\Xi_{i}\right)} \leq \underset{\Xi_{i}}{\operatorname{osc}} f+\underset{\Xi_{i}}{\operatorname{osc}} \operatorname{div} \mathbf{A}\left\|\nabla u_{\mathscr{T}}\right\|_{L^{\infty}\left(\Xi_{i}\right)}+C\left\|\llbracket \mathbf{A} \cdot \nabla u_{\mathscr{G}} \mathbb{\|}\right\|_{L^{\infty}\left(\Lambda_{i}\right)} .
$$

Therefore, invoking (4.19), we see that

$$
\begin{aligned}
& \max _{T \subset \Xi_{i}} h_{T}^{2}\left|R\left(\mathbf{x}_{T}\right)\right| \leq C h_{i}^{2}\left\|R-R_{i}\right\|_{L^{\infty}\left(\Xi_{i}\right)}+C h_{i}\left\|\llbracket \mathbf{A} \cdot \nabla u_{\mathscr{G}} \rrbracket\right\|_{L^{\infty}\left(\Lambda_{i}\right)} \\
& \quad \leq C h_{\mathscr{I}}^{2}\left(\sigma_{f}\left(h_{\mathscr{T}}\right)+h_{\mathscr{G}}^{\alpha}+\left\|e_{\mathscr{T}}\right\|_{L^{\infty}(\Omega)}\right)+C \max _{S \subset \Lambda_{i}}\left(h_{S}\left\|\llbracket \mathbf{A} \cdot \nabla u_{\mathscr{T}} \rrbracket\right\|_{L^{\infty}(S)}\right) .
\end{aligned}
$$

In view of (4.14), Theorem 4.2 and the assumption on $\sigma_{f}$, we end up with

$$
C h_{\mathscr{I}}^{2} \leq\left\|e_{\mathscr{T}}\right\|_{L^{\infty}(\Omega)} \leq o\left(h_{\mathscr{T}}^{2}\right)+C h_{\mathscr{I}}^{2}\left|\log h_{\mathscr{T}}\right|^{2}\left\|e_{\mathscr{G}}\right\|_{L^{\infty}(\Omega)}+C\left|\log h_{\mathscr{T}}\right|^{2} \mathscr{E}_{\mathscr{g}}^{3},
$$

which yields the upper bound in (5.1). The lower bound follows from $\mathscr{E}_{\mathscr{g}}^{3} \leq$ $C \mathscr{E}_{\mathscr{g}}^{2}$.

Remark 5.1. The rightmost term in (5.1) is very reminiscent of the usual a priori error estimate in the maximum norm

$$
\left\|e_{\mathscr{g}}\right\|_{L^{\infty}(\Omega)} \leq C\left|\log h_{\mathscr{T}}\right|^{2.5} \max _{T \in \mathscr{T}}\left(h_{T}^{2}\left\|D^{2} u\right\|_{L^{\infty}(T)}\right)
$$

valid for convex $\Omega$ and mildly graded meshes, that is, those meshes satisfying $|\nabla h(\mathbf{x})| \ll 1, h(\mathbf{x})$ being a mesh density function [11]. This result is used in [12] as an alternative to a lower bound to assess efficiency. A pointwise analysis of the pollution effect of reentrant corners was carried out in [23], where an estimate slightly weaker than (5.4) was derived for meshes exhibiting radial symmetry in the vicinity of corners.

Remark 5.2. The upper bound in (5.1) may be viewed as resulting from replacing formally $D^{2} u$ in (5.4) by the discrete second derivatives $D_{S}^{2} u_{\mathscr{T}}=$ $\left|\llbracket \nabla u_{\mathscr{T}} \rrbracket_{S}\right| / h_{S}$. Such an interpretation was crucial in $[10,13]$ in studying an adaptive procedure for radially symmetric singularities.

\section{CASE $\mathbf{g} \neq 0$ : LiNe Singularities}

Our aim now is to study the effect of a line singularity $\Gamma$ of $\mathbf{g}$. We first discuss the case of a curve $\Gamma$ aligned with $\mathscr{T}$, which divides $\Omega$ into two disjoint polygons $\Omega_{1}$ and $\Omega_{2}$.

Theorem 6.1. Let $\Gamma$ be a polygonal made of sides of $\mathscr{S}$. Let $\mathbf{A} \in\left[W^{2, \infty}(\Omega)\right]^{4}$, and let the modulus of continuity of $f$ in the entire $\Omega$, and that of $\nabla \mathbf{g}$ in $\Omega_{1}$ and $\Omega_{2}$, satisfy $\sigma_{f}(t), \sigma_{\nabla \mathbf{g}}(t)=o\left(|\log t|^{-2}\right)$. If (4.14) holds, then there exist constants $C_{1}, C_{2}, h^{*}>0$ independent of $u$ and $\mathscr{T}$ such that

$$
C_{1} \mathscr{E}_{\mathscr{g}}^{4} \leq\left\|e_{\mathscr{g}}\right\|_{L^{\infty}(\Omega)} \leq C_{2}\left|\log h_{\mathscr{T}}\right|^{2} \mathscr{E}_{\mathscr{g}}^{4} \quad \forall h_{\mathscr{T}} \leq h^{*} .
$$

Proof. We proceed as in Theorem 5.1 and use the same notation. Let $\operatorname{div} g$ denote the elementwise divergence operator, and let the residual $R$ be $R:=$ $f+\operatorname{div}_{\mathscr{G}}\left(\mathbf{A} \cdot \nabla u_{\mathcal{G}}+\mathbf{g}\right)$. Then

$$
\left|R_{i}\right| \leq\left\|R-R_{i}\right\|_{L^{\infty}\left(\Xi_{i}\right)}+C h_{i}^{-1}\left\|\llbracket \mathbf{A} \cdot \nabla u_{\mathscr{T}}+\mathbf{g} \rrbracket\right\|_{L^{\infty}\left(\Lambda_{i}\right)}
$$


On adding and subtracting $I_{\mathscr{g}} \mathbf{A}$ and $\Pi_{\mathscr{g}} \mathbf{g}$, and using (2.11) for $I_{\mathscr{g}}$ and $\Pi_{\mathscr{g}}$, we readily get

$$
\begin{aligned}
& \left\|R-R_{i}\right\|_{L^{\infty}\left(\Xi_{i}\right)} \\
& \leq C\left\|\operatorname{div}\left(\mathbf{A}-I_{\mathscr{G}} \mathbf{A}\right)\right\|_{L^{\infty}\left(\Xi_{i}\right)}\left\|\nabla u_{\mathscr{G}}\right\|_{L^{\infty}\left(\Xi_{i}\right)}+C\left\|\operatorname{div}_{\mathscr{G}}\left(\mathbf{g}-\Pi_{\mathscr{G}} \mathbf{g}\right)\right\|_{L^{\infty}\left(\boldsymbol{\Xi}_{i}\right)} \\
& +\underset{\Xi_{i}}{\operatorname{osc}} f+\left\|\operatorname{div}_{\mathscr{G}}\left(I_{\mathscr{G}} \mathbf{A} \cdot \nabla u_{\mathscr{G}}+\Pi_{\mathscr{g}} \mathbf{g}\right)-\left.\operatorname{div}\left(I_{\mathscr{G}} \mathbf{A} \cdot \nabla u_{\mathscr{G}}+\Pi_{\mathscr{g}} \mathbf{g}\right)\right|_{T_{i}}\right\|_{L^{\infty}\left(\Xi_{i}\right)} \\
& \leq o\left(\left|\log h_{\mathscr{G}}\right|^{-2}\right)+C h_{i}\left\|\nabla u_{\mathscr{G}}\right\|_{L^{\infty}\left(\Xi_{i}\right)}+C h_{i}^{-1}\left\|\llbracket \mathbf{A} \cdot \nabla u_{\mathscr{G}}+\mathbf{g} \rrbracket\right\|_{L^{\infty}\left(\Xi_{i}\right)} .
\end{aligned}
$$

Here we have used an inverse inequality to eliminate $\operatorname{div}_{\mathscr{g}}$ in the second line, and then reason as in (5.3) with $I_{\mathscr{G}} \mathbf{A} \cdot \nabla u_{\mathscr{G}}+\Pi_{\mathscr{g}} \mathbf{g}$. The argument concludes as in Theorem 5.1.

We consider now a line discontinuity $\Gamma$ which is not necessarily aligned with $\mathscr{T}$. Suppose in the sequel that $f$ and $\mathbf{A}$ are as in Theorem 5.1, $\Gamma$ is a Lipschitz curve that divides $\Omega$ into two disjoint domains $\Omega_{1}$ and $\Omega_{2}, n_{\Gamma}$ is the unit normal to $\Gamma$ pointing toward $\Omega_{1}$, and $\mathbf{g}$ satisfies

$$
\mathbf{g} \in W^{1, \infty}\left(\Omega_{1}\right) \cap W^{1, \infty}\left(\Omega_{2}\right), \quad 0<C^{*} \leq\left|\llbracket \mathbf{g}\left\|_{\Gamma}|\leq C| \llbracket \mathbf{g}\right\|_{\Gamma} \cdot \mathbf{n}_{\Gamma}\right| .
$$

This nondegeneracy jump condition means that the line singularity possesses a uniform strength, but it will only be used in the vicinity of a point where the error attains the maximum norm. The best possible (classical) regularity result is expressed by

$$
u \in C^{1, \alpha}\left(\overline{\Omega_{1}}\right) \cap C^{1, \alpha}\left(\overline{\Omega_{2}}\right), \quad \mathbf{A} \cdot \llbracket \nabla u \rrbracket_{\Gamma} \cdot \mathbf{n}_{\Gamma}=-\llbracket \mathbf{g} \rrbracket_{\Gamma} \cdot \mathbf{n}_{\Gamma} \neq 0 .
$$

Suppose $\Gamma_{T}:=\Gamma \cap T \neq \varnothing$ for some $T \in \mathscr{T} ; \Gamma_{T}$ may contain part of $\partial T$. In the next two lemmas we compare the relative size of the various summands in (4.5).

Lemma 6.1. The following estimate is valid provided $\Gamma_{T} \neq \varnothing$ :

$$
\begin{aligned}
\left\|\mathbf{g}-\Pi_{\mathscr{G}} \mathbf{g}\right\|_{L^{\infty}(T)}+h_{T} \| f & +\operatorname{div} \mathbf{A} \cdot \nabla u_{\mathscr{T}}+\operatorname{div} \Pi_{\mathscr{T}} \mathbf{g} \|_{L^{\infty}(T)} \\
& +\left\|\llbracket \Pi_{\mathcal{G}} \mathbf{g} \rrbracket\right\|_{L^{\infty}(\partial T)} \leq C\left\|\llbracket \mathbf{g} \rrbracket_{\Gamma}\right\|_{L^{\infty}\left(\Gamma_{T}\right)} .
\end{aligned}
$$

Proof. By virtue of (2.10) and (6.2), we deduce that

$$
\left\|\mathbf{g}-\Pi_{\mathscr{G}} \mathbf{g}\right\|_{L^{\infty}(T)} \leq \underset{T}{\operatorname{osc}}|\mathbf{g}| \leq\left\|\llbracket \mathbf{g} \rrbracket_{\Gamma}\right\|_{L^{\infty}\left(\Gamma_{T}\right)}+C h_{T} \leq C\left\|\llbracket \mathbf{I g} \rrbracket_{\Gamma}\right\|_{L^{\infty}\left(\Gamma_{T}\right)} .
$$

On the other hand, since $\operatorname{div} \Pi_{\mathscr{g}} \mathbf{g} \in \mathscr{P}_{0}(T)$, we have

$$
\begin{aligned}
C h_{T}^{2} \operatorname{div} \Pi_{\mathscr{G}} \mathbf{g} & =\left\langle\operatorname{div} \Pi_{\mathscr{G}} \mathbf{g}, 1\right\rangle_{T}=\left\langle\left\langle\Pi_{\mathscr{G}} \mathbf{g}, 1\right\rangle\right\rangle_{\partial T} \\
& =\langle\langle\mathbf{g}, 1\rangle\rangle_{\partial T}=\langle\operatorname{div} \mathbf{g}, 1\rangle_{T \backslash \Gamma}+\left\langle\left\langle\llbracket \mathbf{I} \rrbracket_{\Gamma} \cdot \mathbf{n}_{\Gamma}, 1\right\rangle\right\rangle_{\Gamma_{T}} .
\end{aligned}
$$

In view of (6.1), we see that $\left|\langle\operatorname{div} \mathbf{g}, 1\rangle_{T \backslash \Gamma}\right| \leq C h_{T}^{2}$, whence

$$
h_{T}\left|\operatorname{div} \Pi_{\mathscr{g}} \mathbf{g}\right| \leq C h_{T}+C\left\|\llbracket \mathbf{g} \rrbracket_{\Gamma}\right\|_{L^{\infty}\left(\Gamma_{T}\right)} \leq C\left\|\llbracket \mathbf{g} \rrbracket_{\Gamma}\right\|_{L^{\infty}\left(\Gamma_{T}\right)} .
$$

Also

$$
\left\|\llbracket \Pi_{g} \mathbf{g} \rrbracket\right\|_{L^{\infty}(\partial T)} \leq C\|\llbracket \mathbf{g} \rrbracket\|_{L^{\infty}(\Gamma \cap \partial T)},
$$

as results from (2.10). Since $h_{T}\|f\|_{L^{\infty}(T)} \leq C h_{T}$ is asymptotically negligible, as compared with $\left\|\llbracket \mathbf{g} \rrbracket_{\Gamma}\right\|_{L^{\infty}\left(\Gamma_{T}\right)}$, it only remains to demonstrate that so is $h_{T}\left\|\operatorname{div} \mathbf{A} \cdot \nabla u_{\mathcal{G}}\right\|_{L^{\infty}(T)}$. To this end, we recall that $u \in H^{1+\varepsilon}(\Omega)$ for some $\varepsilon>0$ because $f+\operatorname{div} \mathbf{g} \in H^{-1+\varepsilon}(\Omega)$ [9]. As a consequence of Sobolev's imbedding 
theorem we also have $u \in W^{1, p}(\Omega)$ for some $p>2$. By virtue of standard a priori error analysis in $H_{0}^{1}(\Omega)$, and Hölder's inequality, we arrive at

$$
\begin{aligned}
h_{T}\left\|\operatorname{div} \mathbf{A} \cdot \nabla u_{\mathscr{G}}\right\|_{L^{\infty}(T)} & \leq C\left\|\nabla u_{\mathscr{G}}\right\|_{L^{2}(T)} \leq C\left\|\nabla e_{\mathscr{G}}\right\|_{L^{2}(T)}+C\|\nabla u\|_{L^{2}(T)} \\
& \leq C h_{\mathscr{g}}^{\varepsilon}\|u\|_{H^{1+\varepsilon}(\Omega)}+C h_{\mathscr{g}}^{(p-2) / p}\|\nabla u\|_{L^{p}(\Omega)}=o(1) .
\end{aligned}
$$

This concludes the proof.

Set $\Gamma_{i}:=\Gamma \cap \Xi_{i}$ and denote by $h_{i}$ the size of $\Xi_{i}$. Note that the nondegeneracy property

$$
\operatorname{dist}\left(\Gamma_{i}, \partial \Xi_{i}\right) \geq C h_{i}
$$

for some $0<C<1 / 2$ is equivalent to assuming that $\Gamma$ splits $\Xi_{i}$ into two comparable pieces.

Lemma 6.2. If (6.5) holds, then $\left\|\llbracket \mathbf{I g} \rrbracket_{\Gamma} \cdot \mathbf{n}_{\Gamma}\right\|_{L^{\infty}\left(\Gamma_{i}\right)} \leq C\left\|\llbracket \mathbf{A} \cdot \nabla u_{g} \mathbb{\rrbracket}\right\|_{L^{\infty}\left(\Lambda_{i}\right)}$.

Proof. Let $\mathbf{x}_{k} \in \Xi_{i} \cap \Omega_{k}$ be fixed and set $\mathbf{g}_{k}:=\left.\mathbf{g}\right|_{\Omega_{k}}$ for $k=1$, 2. Upon integration by parts, we can write the discrete equation in (2.6) as follows:

$$
\begin{aligned}
\left\langle\left\langle\mathbf{g}\left(\mathbf{x}_{1}\right)-\mathbf{g}\left(\mathbf{x}_{2}\right),\right.\right. & \left.\left.\mathbf{n}_{\Gamma} \varphi_{i}\right\rangle\right\rangle_{\Gamma_{i}}=-\left\langle\left\langle\llbracket \mathbf{A} \cdot \nabla u_{\mathscr{G}} \rrbracket, \varphi_{i}\right\rangle\right\rangle_{\Lambda_{i}}-\left\langle\operatorname{div} \mathbf{A} \cdot \nabla u_{\mathscr{T}}, \varphi_{i}\right\rangle_{\Xi_{i}} \\
& +\left\langle\left\langle\left(\mathbf{g}\left(\mathbf{x}_{1}\right)-\mathbf{g}_{1}\right)-\left(\mathbf{g}\left(\mathbf{x}_{2}\right)-\mathbf{g}_{2}\right), \mathbf{n}_{\Gamma} \varphi_{i}\right\rangle\right\rangle_{\Gamma_{i}}-\left\langle f+\operatorname{div} \mathbf{g}, \varphi_{i}\right\rangle_{\left.\Xi_{i}\right\rangle \Gamma} .
\end{aligned}
$$

By virtue of (2.2) and (6.2), the two rightmost terms of the right-hand side are $\leq C h_{i}^{2}$. The second term on the right-hand side is, instead, of order $o\left(h_{\mathscr{g}}\right)$ because of (6.4). Hence, since (6.5) yields $\int_{\Gamma_{i}} \varphi_{i} \geq C h_{i}$, we have

$$
\left|\left(\mathbf{g}\left(\mathbf{x}_{1}\right)-\mathbf{g}\left(\mathbf{x}_{2}\right)\right) \cdot \mathbf{n}_{\Gamma}\right| \leq C\left\|\llbracket \mathbf{A} \cdot \nabla u_{\mathscr{G}} \mathbb{I}\right\|_{L^{\infty}\left(\boldsymbol{\Lambda}_{i}\right)}+o(1) .
$$

Therefore,

$$
\begin{aligned}
& C \leq\left\|\mathbb{I} \mathbf{g} \rrbracket_{\Gamma} \cdot \mathbf{n}_{\Gamma}\right\|_{L^{\infty}\left(\Gamma_{i}\right)} \\
& \leq C\left\|\llbracket \mathbf{A} \cdot \nabla u_{g} \rrbracket\right\|_{L^{\infty}\left(\Lambda_{i}\right)}+\left\|\left[\left(\mathbf{g}\left(\mathbf{x}_{1}\right)-\mathbf{g}_{1}\right)-\left(\mathbf{g}\left(\mathbf{x}_{2}\right)-\mathbf{g}_{2}\right)\right] \cdot \mathbf{n}_{\Gamma}\right\|_{L^{\infty}\left(\Gamma_{i}\right)}+o(1) \\
& \leq C\left\|\llbracket \mathbb{A} \cdot \nabla u_{\mathscr{G}} \mathbb{\rrbracket}\right\|_{L^{\infty}\left(\Lambda_{i}\right)}+o(1) \leq C\left\|\llbracket \mathbf{A} \cdot \nabla u_{\mathscr{G}} \mathbb{\rrbracket}\right\|_{L^{\infty}\left(\Lambda_{i}\right)} \text {. }
\end{aligned}
$$

We are now in a position to derive an upper bound for the pointwise error. To this end, we need another nondegeneracy assumption on $\Gamma$, namely that $\Gamma$ does not intersect $\partial \Omega$ tangentially. For each $\mathbf{x}_{0} \in \Gamma \cap \partial \Omega$, this entails the existence of a closed truncated cone $\mathscr{C}_{\mathbf{x}_{0}}$ of center $\mathbf{x}_{0}$ and height $r$ such that

$$
\left\{\mathbf{x} \in \Gamma:\left|\mathbf{x}_{0}-\mathbf{x}\right| \leq r\right\} \subset \mathscr{C}_{\mathbf{x}_{0}} \subset \Omega .
$$

Theorem 6.2. Let $\mathbf{A} \in\left[W^{2, \infty}(\Omega)\right]^{4}$, and let $f$ and $\nabla \mathbf{g}$ be uniformly continuous in $\Omega_{1}$ and $\Omega_{2}$ with moduli of continuity $\sigma_{f}(t), \sigma_{\nabla \mathbf{g}}(t)=o\left(|\log t|^{-2}\right)$. If both (4.14) and (6.6) are valid, then there exist $C, h^{*}>0$ independent of $u$ and $\mathscr{T}$ such that

$$
\left\|e_{\mathscr{G}}\right\|_{L^{\infty}(\Omega)} \leq C\left|\log h_{\mathscr{I}}\right|^{2} \max _{S \in \mathscr{S}}\left(h_{S}\left\|\llbracket \mathbf{A} \cdot \nabla u_{\mathscr{G}} \mathbb{\rrbracket}\right\|_{L^{\infty}(S)}\right) \quad \forall h_{\mathscr{T}} \leq h^{*} .
$$

Proof. Let $T_{0} \in \mathscr{T}$ satisfy $E_{T_{0}}^{1}=\mathscr{E}_{\mathscr{g}}$. If $\Gamma \cap T=\varnothing$ for all adjacent elements $T$ to $T_{0}$, then we can argue as in Theorem 5.1 to arrive at $E_{T_{0}}^{1} \leq C \mathscr{E}_{g}^{3}$. Otherwise, there exists an adjacent element $T_{1}$, possibly $T_{0}$ itself, satisfying $T_{1} \cap \Gamma \neq \varnothing$. Let $\mathbf{x}_{i}$ be a vertex of $T_{1}$ closest to $T_{1} \cap \Gamma$. If $\mathbf{x}_{i}$ is an interior node, then $\Xi_{i}$ satisfies (6.5). If $\mathbf{x}_{i} \in \partial \Omega$ instead, then (6.6) yields the existence 
of a set $\Xi_{j} \ni \mathbf{x}_{i}$ satisfying also (6.5). In either case, Lemma 6.2 applies and, together with (6.2), Lemma 6.1 and the fact that $h_{T_{0}} \approx h_{i} \approx h_{j}$ imply

$$
\mathscr{E}_{\mathscr{G}}^{1}=E_{T_{0}}^{1} \leq C h_{T_{0}}\|\llbracket \mathbf{g} \rrbracket\|_{L^{\infty}\left(\Gamma_{i} \cup \Gamma_{j}\right)}+h_{T_{0}}\left\|\llbracket \nabla u_{\mathscr{g}} \rrbracket\right\|_{L^{\infty}\left(\Lambda_{i} \cup \Lambda_{j}\right)} \leq C \mathscr{E}_{\mathscr{G}^{3}}^{3} .
$$

The assertion then follows from Lemma 4.1.

The following result shows that overestimation is quite unlikely whenever the interface $\Gamma$ splits $T$ into two comparable pieces.

Lemma 6.3. If $\operatorname{dist}\left(\Gamma_{T}, \partial T\right) \geq C h_{T}$, then $\left\|e_{g}\right\|_{L^{\infty}(T)} \geq C h_{T}\left\|\llbracket \mathbf{g} \rrbracket_{\Gamma}\right\|_{L^{\infty}\left(\Gamma_{T}\right)}$. Proof. With the aid of (6.3) and interpolation theory, we can write

$$
\begin{aligned}
\left\|e_{\mathscr{G}}\right\|_{L^{\infty}(T)} & \geq C\left\|u-I_{\mathscr{G}} u\right\|_{L^{\infty}(T)} \\
& \geq C \operatorname{dist}\left(\Gamma_{T}, \partial T\right)\|\mathbf{I g}\|_{\Gamma}\left\|_{L^{\infty}\left(\Gamma_{T}\right)} \geq C h_{T}\right\| \llbracket \mathbf{g}\left\|_{\Gamma}\right\|_{L^{\infty}\left(\Gamma_{T}\right)} .
\end{aligned}
$$

Remark 6.1. The most difficult situation, not covered by the above analysis, is that of an interface $\Gamma$ being almost parallel to a side and very close to it. In such a case, the error might be much smaller than first order. This issue warrants further research.

Remark 6.2. The estimator $\mathscr{E}_{\mathscr{G}}^{3}$ has been already used successfully for the adaptive solution of time-dependent free boundary problems with $\mathbf{A}=\mathbf{I}$ [21].

\section{EQuivaleNT ESTIMATORS}

The purpose of this last section is to prove that other local a posteriori estimators, typically used in connection with the energy norm, provide also information to estimate the pointwise error $[6,24,25]$. We in fact show that these estimators, when properly interpreted, are equivalent to $\left\|e_{\mathcal{G}}\right\|_{L^{\infty}(\Omega)}$. The estimators in $[6,24]$ are based on solving local problems, whereas that in [25] consists of an averaging postprocessing or gradient recovery. For simplicity, we assume $\mathbf{A}=\mathbf{I}$ and $\mathbf{g}=0$.

7.1. Verfürth's estimator. Let $\mathscr{P}_{2}^{0}(T)$ denote the set of quadratic polynomials in $T \in \mathscr{T}$ that vanish at the vertices of $T$. Let $\mathscr{U}_{T}$ indicate the direct sum of $\mathscr{P}_{2}^{0}(T)$ and the space of cubic bubbles. Let $\omega_{T} \in \mathscr{U}_{T}$ satisfy

$$
\left\langle\nabla \omega_{T}, \nabla \varphi\right\rangle_{T}=\left\langle f\left(\mathbf{x}_{T}\right), \varphi\right\rangle_{T}+\frac{1}{2}\left\langle\left\langle\llbracket \nabla u_{\mathscr{T}} \rrbracket_{S}, \varphi\right\rangle_{\partial T} \quad \forall \varphi \in \mathscr{U}_{T} .\right.
$$

Note that this is just a modification of the estimator introduced by Bank and Weiser [6], for which $\mathscr{U}_{T}=\mathscr{P}_{2}^{0}(T)$. The presence of the extra bubble functions enables us to prove the desired equivalence result under least restrictive regularity assumptions.

Corollary 7.1. Let the modulus of continuity of $f$ satisfy $\sigma_{f}(t)=o\left(|\log t|^{-2}\right)$ within each finite element, and let $\left\|e_{\mathscr{g}}\right\|_{L^{\infty}(\Omega)} \geq C h_{\mathscr{G}}^{2}$. Then there exist $C_{1}, C_{2}$, $h^{*}>0$ independent of $u$ and $\mathscr{T}$ such that for all $h_{\mathscr{T}} \leq h^{*}$

$$
C_{1} \max _{T \in \mathscr{T}}\left\|\omega_{T}\right\|_{L^{\infty}(T)} \leq\left\|e_{\mathscr{G}}\right\|_{L^{\infty}(\Omega)} \leq C_{2}\left|\log h_{\mathscr{T}}\right|^{2} \max _{T \in \mathscr{T}}\left\|\omega_{T}\right\|_{L^{\infty}(T)} .
$$

Proof. We proceed now to show the equivalence of $\max _{T \in \mathcal{G}}\left\|\omega_{T}\right\|_{L^{\infty}(T)}$ and $\mathscr{E}_{\mathscr{g}}^{2}$. The first simple observation is that

$$
\|\varphi\|_{L^{\infty}(T)} \approx h_{T}\|\nabla \varphi\|_{L^{\infty}(T)} \approx\|\nabla \varphi\|_{L^{2}(T)} \quad \forall \varphi \in \mathscr{U}_{T},
$$


because $\varphi$ is piecewise cubic and vanishes at the vertices of $T$. Taking $\varphi=\omega_{T}$ in (7.1), and using (7.3) in conjunction with Theorem 4.2, yields

$$
\begin{aligned}
\left\|\omega_{T}\right\|_{L^{\infty}(T)}^{2} & \leq C\left\|\nabla \omega_{T}\right\|_{L^{2}(T)}^{2} \\
& \leq C\left(h_{T}^{2}\left|f\left(\mathbf{x}_{T}\right)\right|+h_{T}\left\|\llbracket \nabla u_{g} \rrbracket\right\|_{L^{\infty}(\partial T)}\right)\left\|\omega_{T}\right\|_{L^{\infty}(T)} \\
& =C E_{T}^{2}\left\|\omega_{T}\right\|_{L^{\infty}(T)} \leq C \mathscr{E}_{g}^{2}\left\|\omega_{T}\right\|_{L^{\infty}(T)} .
\end{aligned}
$$

In order to prove the reverse inequality, we argue as in Lemma 4.2. Let $b_{T} \in$ $\mathscr{P}_{3}(T)$ be the canonical bubble function of $T$, and $\left\{\varphi_{S}\right\}$ be the canonical basis of $\mathscr{P}_{2}^{0}(T)$. Let $\varphi=\alpha_{T} b_{T}+\sum_{S \subset \partial T} \beta_{S} \varphi_{S} \in \mathscr{U}_{T}$ be defined by

$$
\begin{gathered}
\beta_{S}\left\langle\left\langle\mathbb{\amalg} \nabla u_{\mathscr{G}} \mathbb{1}_{S}, \varphi_{S}\right\rangle_{S}=2 h_{S}\left|\llbracket \nabla u_{\mathscr{T}} \mathbb{\rrbracket}_{S}\right|,\right. \\
\alpha_{T}\left\langle f\left(\mathbf{x}_{T}\right), b_{T}\right\rangle_{T}=h_{T}^{2}\left|f\left(\mathbf{x}_{T}\right)\right|-\sum_{S \subset \partial T} \beta_{S}\left\langle f\left(\mathbf{x}_{T}\right), \varphi_{S}\right\rangle,
\end{gathered}
$$

which leads to $\left|\alpha_{T}\right|,\left|\beta_{S}\right| \leq C$, because $\llbracket \nabla u_{g} \rrbracket_{S}$ and $f\left(\mathbf{x}_{T}\right)$ are constants. Therefore, by virtue of (7.1) and (7.3), we see that

$$
\begin{aligned}
E_{T}^{2} & \leq h_{T}^{2}\left|f\left(\mathbf{x}_{T}\right)\right|+\sum_{S \subset \partial T} h_{S}\left|\mathbb{L} \nabla u_{g} \mathbb{I}_{S}\right|=\left\langle f\left(\mathbf{x}_{T}\right), \varphi\right\rangle_{T}+\frac{1}{2}\left\langle\left\langle\llbracket \nabla u_{\mathcal{G}} \mathbb{\rrbracket}_{S}, \varphi\right\rangle_{\partial T}\right. \\
& =\left\langle\nabla \omega_{T}, \nabla \varphi\right\rangle_{T} \leq C\left\|\omega_{T}\right\|_{L^{\infty}(T)}\|\varphi\|_{L^{\infty}(T)} \leq C\left\|\omega_{T}\right\|_{L^{\infty}(T)} .
\end{aligned}
$$

We point out that this proof shows the equivalence of $\left\|\omega_{T}\right\|_{L^{\infty}(T)}$ and $E_{T}^{2}$ at the element level, even for $f$ with jump discontinuities across interelement boundaries. Such equivalence might fail to hold if we suppress the bubbles as in [6].

7.2. Bank-Weiser's estimator. Let $\xi_{T} \in \mathscr{P}_{2}^{0}(T)$ be the solution of (7.1) for all $\varphi \in \mathscr{P}_{2}^{0}(T)$ [6]. So we now have one fewer degree of freedom with respect to $\mathscr{U}_{T}$. But to demonstrate equivalence, we compensate with global continuity of $f$.

Corollary 7.2. Let the modulus of continuity of $f$ in the entire $\Omega$ satisfy $\sigma_{f}(t)=$ $o\left(|\log t|^{-2}\right)$, and let $\left\|e_{\mathscr{g}}\right\|_{L^{\infty}(\Omega)} \geq C h_{\mathscr{G}}^{2}$. Then there exist $C_{1}, C_{2}, h^{*}>0$ independent of $u$ and $\mathscr{T}$ such that for all $h_{\mathscr{G}} \leq h^{*}$

$$
C_{1} \max _{T \in \mathscr{T}}\left\|\xi_{T}\right\|_{L^{\infty}(T)} \leq\left\|e_{\mathscr{G}}\right\|_{L^{\infty}(\Omega)} \leq C_{2}\left|\log h_{\mathscr{G}}\right|^{2} \max _{T \in \mathscr{T}}\left\|\xi_{T}\right\|_{L^{\infty}(T)} .
$$

Proof. Arguing as in Corollary 7.1, we get $\left\|\xi_{T}\right\|_{L^{\infty}(T)} \leq C E_{T}^{2} \leq C\left\|e_{\mathscr{G}}\right\|_{L^{\infty}(\Omega)}$ because of (4.16). To derive the reverse inequality, we cannot proceed elementwise as before, but rather we have to deal with the set $\Xi_{i}$ associated with a generic node $\mathbf{x}_{i}$ as in Theorem 5.1. Consider the piecewise quadratic function $\varphi=\sum_{S \subset \Lambda_{i}} \beta_{S} \varphi_{S}$, with $\beta_{S}$ defined as in (7.5); thus $\|\varphi\|_{L^{\infty}\left(\Xi_{i}\right)} \leq C$. We can write

$$
\begin{aligned}
2 \sum_{S \subset \Lambda_{i}} h_{S}\left|\mathbb{I} \nabla u_{\mathscr{G}} \mathbb{\rrbracket}_{S}\right|= & \left\langle\left\langle\llbracket \nabla u_{\mathscr{G}} \mathbb{\rrbracket}, \varphi\right\rangle\right\rangle_{\Lambda_{i}} \\
= & \sum_{T \subset \Xi_{i}}\left(\left\langle f\left(\mathbf{x}_{T}\right), \varphi\right\rangle_{T}+\frac{1}{2}\left\langle\left\langle\llbracket \nabla u_{\mathscr{G}} \mathbb{\rrbracket}, \varphi\right\rangle_{\partial T}\right)\right. \\
& -\left\langle f\left(\mathbf{x}_{i}\right), \varphi\right\rangle_{\Xi_{i}}+\sum_{T \subset \Xi_{i}}\left\langle f\left(\mathbf{x}_{i}\right)-f\left(\mathbf{x}_{T}\right), \varphi\right\rangle_{T}=: I+I I+I I I,
\end{aligned}
$$


and examine each term separately. In light of (7.3), we first have

$$
|I| \leq \sum_{T \subset \Xi_{i}}\left|\left\langle\nabla \xi_{T}, \nabla \varphi\right\rangle_{T}\right| \leq C \max _{T \subset \Xi_{i}}\left(\left\|\xi_{T}\right\|_{L^{\infty}(T)}\|\varphi\|_{L^{\infty}(T)}\right) \leq C \max _{T \subset \Xi_{i}}\left\|\xi_{T}\right\|_{L^{\infty}(\Omega)} .
$$

Utilizing (5.2) and properties $\left\langle\varphi_{i}, 1\right\rangle_{\Xi_{i}}=\left|\Xi_{i}\right| / 3$ and $\left\langle\left\langle\varphi_{i}, 1\right\rangle\right\rangle_{S}=h_{S} / 2$, we deduce that

$$
\begin{aligned}
\left|f\left(\mathbf{x}_{i}\right)\right| & \leq \frac{1}{\left\langle\varphi_{i}, 1\right\rangle_{\Xi_{i}}} \mid \sum_{S \subset \Lambda_{i}} \llbracket \nabla u_{\mathscr{G}} \rrbracket_{s}\left\langle\left\langle\varphi_{i}, 1\right\rangle_{S}\right|+\left\|f\left(\mathbf{x}_{i}\right)-f\right\|_{L^{\infty}\left(\Xi_{i}\right)} \\
& \leq \frac{3}{2\left|\Xi_{i}\right|}\left|\sum_{S \subset \Lambda_{i}} h_{S} \llbracket \nabla u_{\mathscr{G}} \rrbracket_{S}\right|+\sigma_{f}\left(h_{i}\right) .
\end{aligned}
$$

Hence,

$$
|I I| \leq\left|f\left(\mathbf{x}_{i}\right)\right|\|\varphi\|_{L^{\infty}\left(\Xi_{i}\right)}\left|\Xi_{i}\right| \leq C h_{i}^{2} \sigma_{f}\left(h_{i}\right)+C\left|\sum_{S \subset \Lambda_{i}} h_{S} \llbracket \nabla u_{\mathscr{G}} \rrbracket s\right| .
$$

Since $|I I I| \leq C h_{i}^{2} \sigma_{f}\left(h_{i}\right)$, we are led to evaluate the contribution of the rightmost term in the preceding inequality. Note that, as compared with the original expression, the absolute values are now outside the summation. This fact will be exploited in the sequel.

To do so, we introduce the piecewise quadratic function $\zeta=\sum_{S \subset \Lambda_{i}} \varphi_{S}$. Given $S \in \mathscr{S}$, let $T_{S}$ stand for the union of the adjacent triangles of $\mathscr{T}$ sharing $S$, and let $\left|T_{S}\right|$ denote its measure. We see that

$$
\left\langle f\left(\mathbf{x}_{i}\right), \zeta\right\rangle=\sum_{S \subset \Lambda_{i}}\left\langle f\left(\mathbf{x}_{i}\right), \varphi_{S}\right\rangle=\sum_{S \subset \Lambda_{i}} \frac{\left|T_{S}\right|}{3} f\left(\mathbf{x}_{i}\right)=\frac{2}{3} f\left(\mathbf{x}_{i}\right)\left|\Xi_{i}\right|=2\left\langle f\left(\mathbf{x}_{i}\right), \varphi_{i}\right\rangle,
$$

because $\left\langle\varphi_{S}, 1\right\rangle=\left|T_{S}\right| / 3$. Therefore, since $\left\langle\left\langle\varphi_{S}, 1\right\rangle\right\rangle_{S}=\frac{4}{3}\left\langle\left\langle\varphi_{i}, 1\right\rangle\right\rangle_{S}$, we get

$$
\begin{aligned}
\sum_{T \subset \Xi_{i}}\left\langle\nabla \xi_{T},\right. & \nabla \zeta\rangle_{T}=\sum_{T \subset \Xi_{i}}\left(\frac{1}{2}\left\langle\left\langle\llbracket \nabla u_{\mathscr{G}} \rrbracket, \zeta\right\rangle_{\partial T}+\left\langle f\left(\mathbf{x}_{T}\right), \zeta\right\rangle_{T}\right)\right. \\
& =\left\langle\left\langle\llbracket \nabla u_{\mathscr{T}} \rrbracket, \zeta\right\rangle_{\Lambda_{i}}+\left\langle f\left(\mathbf{x}_{i}\right), \zeta\right\rangle_{\Xi_{i}}+\sum_{T \subset \Xi_{i}}\left\langle f\left(\mathbf{x}_{T}\right)-f\left(\mathbf{x}_{i}\right), \zeta\right\rangle_{T}\right. \\
& =\frac{4}{3}\left\langle\left\langle\llbracket \nabla u_{\mathscr{T}} \rrbracket, \varphi_{i}\right\rangle\right\rangle_{\Lambda_{i}}+2\left\langle f\left(\mathbf{x}_{i}\right), \varphi_{i}\right\rangle_{\Xi_{i}}+\sum_{T \subset \Xi_{i}}\left\langle f\left(\mathbf{x}_{T}\right)-f\left(\mathbf{x}_{i}\right), \zeta\right\rangle_{T} \\
& =-\frac{1}{3} \sum_{S \subset \Lambda_{i}} h_{S} \llbracket \nabla u_{\mathscr{G}} \rrbracket_{S}+2\left\langle f\left(\mathbf{x}_{i}\right)-f, \varphi_{i}\right\rangle_{\Xi_{i}}+\sum_{T \subset \Xi_{i}}\left\langle f\left(\mathbf{x}_{T}\right)-f\left(\mathbf{x}_{i}\right), \zeta\right\rangle_{T},
\end{aligned}
$$

where we have used (5.2) in the last passage. Consequently,

$$
\begin{aligned}
\left|\sum_{S \subset \Lambda_{i}} h_{S} \llbracket \nabla u_{g} \rrbracket s\right| & \leq 3 \sum_{T \subset \Xi_{i}}\left|\left\langle\nabla \xi_{T}, \nabla \zeta\right\rangle_{T}\right|+C h_{i}^{2} \underset{\Xi_{i}}{\operatorname{osc} f} \\
& \leq C \max _{T \subset \Xi_{i}}\left\|\xi_{T}\right\|_{L^{\infty}(T)}+C h_{i}^{2} \sigma_{f}\left(h_{i}\right) .
\end{aligned}
$$

Upon combining the estimates for $I, I I$, and $I I I$, we realize that

$$
\sum_{S \subset \Lambda_{i}} h_{S}\left|\llbracket \nabla u_{\mathcal{G}} \rrbracket_{S}\right| \leq C \max _{T \subset \Xi_{i}}\left\|\xi_{T}\right\|_{L^{\infty}(T)}+C h_{i}^{2} \sigma_{f}\left(h_{i}\right) .
$$


The nondegeneracy assumption (4.14), together with (5.1), implies

$$
C h_{\mathscr{T}}^{2} \leq C\left|\log h_{\mathscr{I}}\right|^{2} \max _{S \in \mathscr{S}}\left(h_{S}\left|\llbracket \nabla u_{\mathscr{G}} \rrbracket_{S}\right|\right) \leq C\left|\log h_{\mathscr{T}}\right|^{2} \max _{T \in \mathscr{T}}\left\|\xi_{T}\right\|_{L^{\infty}(T)}+o\left(h_{\mathscr{T}}^{2}\right) .
$$

This and (5.1) lead to the conclusion

$$
C\left|\log h_{\mathscr{G}}\right|^{-2}\left\|e_{\mathscr{G}}\right\|_{L^{\infty}(\Omega)} \leq \mathscr{E}_{\mathscr{T}}^{3}=\max _{S \in \mathscr{S}}\left(h_{S}\left|\llbracket \nabla u_{\mathscr{G}} \rrbracket_{S}\right|\right) \leq C \max _{T \in \mathscr{T}}\left\|\xi_{T}\right\|_{L^{\infty}(T)} .
$$

The above proof of equivalence does not rely upon the saturation assumption of [6], which implicitly entails some additional global regularity of $D^{2} u$. In this vein we mention that $\sigma_{f}$ satisfies the Dini condition $\int_{0^{+}} \frac{\sigma_{f}(t)}{t} d t<\infty$, which in turn guarantees the interior continuity of $D^{2} u$; however, $D^{2} u$ blows up at a corner. Consequently, quadratic functions do not provide in general better global approximation than linear ones, thereby making the saturation assumption of [6] fail.

Removing the saturation assumption in the energy norm is also of theoretical importance. The above argument can be modified to achieve such a goal, as shown in [20].

7.3. Zienkiewicz-Zhu's estimator. Consider the following recovered gradient $\mathscr{G}_{\mathscr{g}} \in\left[\mathscr{V}_{\mathscr{g}}^{1} \cap C(\Omega)\right]^{2}$ which, for each node $\mathbf{x}_{i}$, is defined by

$$
\mathscr{G} u_{\mathscr{T}}\left(\mathbf{x}_{i}\right):=\left.\sum_{T \subset \Xi_{i}} \frac{|T|}{\left|\Xi_{i}\right|} \nabla u_{\mathscr{T}}\right|_{T}
$$

Such a postprocessing is simply a weighted average of $\nabla u_{\mathscr{g}}$ over the triangles containing $\mathbf{x}_{i}$. In [25] the computable quantity $\left(\int_{T}\left|\mathscr{G} u_{\mathscr{G}}-\nabla u_{\mathscr{G}}\right|^{2}\right)^{1 / 2}$ is used to estimate the error in energy norm. The following local equivalence result has been recently proved in [22]:

$$
\left.\sum_{T \subset \Xi_{i}}|T|\left|\mathscr{G} u_{\mathscr{G}}\left(\mathbf{x}_{i}\right)-\nabla u_{\mathscr{G}}\right|_{T}\right|^{2} \approx\left|\Xi_{i}\right| \sum_{S \subset \Lambda_{i}}\left|\llbracket \nabla u_{\mathscr{T}} \mathbb{l}_{S}\right|^{2} \text {. }
$$

By virtue of (7.8) it is now easy to demonstrate that the information contained in $\mathscr{G} u_{\mathscr{g}}$ can be used to estimate the pointwise error.

Corollary 7.3. Let the modulus of continuity of $f$ in $\Omega$ satisfy $\sigma_{f}(t)=$ $o\left(|\log t|^{-2}\right)$, and let $C h_{\mathscr{g}}^{2} \leq\left\|e_{\mathscr{G}}\right\|_{L^{\infty}(\Omega)}$. Then there exist $C_{1}, C_{2}, h^{*}>0$ independent of $u$ and $\mathscr{T}$ such that for all $h_{\mathscr{g}} \leq h^{*}$

$$
\begin{aligned}
C_{1} \max _{T \in \mathscr{T}}\left(h_{T}\left\|\mathscr{G} u_{\mathscr{T}}-\nabla u_{\mathscr{G}}\right\|_{L^{\infty}(T)}\right) & \leq\left\|e_{\mathscr{T}}\right\|_{L^{\infty}(\Omega)} \\
& \leq C_{2}\left|\log h_{\mathscr{T}}\right|^{2} \max _{T \in \mathscr{T}}\left(h_{T}\left\|\mathscr{G} u_{\mathscr{G}}-\nabla u_{\mathscr{G}}\right\|_{L^{\infty}(T)}\right) .
\end{aligned}
$$

Proof. It is enough to observe that the minimum angle condition yields

$$
\begin{gathered}
\left.\sum_{T \subset \Xi_{i}}|T|\left|\mathscr{G} u_{\mathscr{G}}\left(\mathbf{x}_{i}\right)-\nabla u_{\mathscr{G}}\right| T\right|^{2} \approx\left(\max _{T \subset \Xi_{i}} h_{T}\left|\mathscr{G} u_{\mathscr{G}}\left(\mathbf{x}_{i}\right)-\nabla u_{\mathscr{G}}\right|_{T} \mid\right)^{2}, \\
\left|\Xi_{i}\right| \sum_{S \subset \Lambda_{i}}\left|\llbracket \nabla u_{\mathscr{G}} \rrbracket_{S}\right|^{2} \approx\left(\max _{S \subset \Lambda_{i}} h_{S}\left|\llbracket \nabla u_{\mathscr{T}} \rrbracket_{S}\right|\right)^{2},
\end{gathered}
$$


and that $\left\|\mathscr{G} u_{\mathscr{G}}-\nabla u_{\mathscr{G}}\right\|_{L^{\infty}(T)}$ is attained at a vertex of $T$, because the underlying function is linear. The assertion finally follows from Theorem 5.1 together with (7.8).

\section{BIBLIOGRAPHY}

1. I. Babuška, R. Durán, and R. Rodriguez, Analysis of the efficiency of an a posteriori error estimator for linear triangular finite elements, SIAM J. Numer. Anal. 29 (1992), 947-964.

2. I. Babuška and A. Miller, $A$ feedback finite element method with a posteriori error estimation: Part I. The finite element method and some basic properties of the a posteriori error estimator, Comput. Methods. Appl. Mech. Engrg. 61 (1987), 1-40.

3. I. Babuška and C. Rheinboldt, Error estimates for adaptive finite element computations, SIAM J. Numer. Anal. 15 (1978), 736-754.

4. I. Babuška, O.C. Zienkiewicz, J. Gago, and E.R. de A. Oliveira, Accuracy estimates and adaptive refinements in finite element computations, Wiley, New York, 1986.

5. R.E. Bank, PLTMG: A software package for solving elliptic partial differential equations, users' guide 6.0, Frontiers in Applied Mathematics, SIAM, Philadelphia, PA, 1990.

6. R.E. Bank and A. Weiser, Some a posteriori error estimators for elliptic partial differential equations, Math. Comp. 44 (1985), 283-301.

7. F. Brezzi, J. Douglas Jr., and L.D. Marini, Two families of mixed finite element methods for second order elliptic problems, Numer. Math. 47 (1985), 217-235.

8. P.G. Ciarlet, The finite element method for elliptic problems, North-Holland, Amsterdam, 1978.

9. M. Dauge, Elliptic boundary value problems on corner domains: smoothness and asymptotics of solutions, Lecture Notes in Math., vol. 1341, Springer-Verlag, Berlin and New York, 1988.

10. K. Eriksson, Adaptive finite element methods based on optimal error estimates for linear elliptic problems, Preprint 1987-02, Chalmers University of Technology, Göteborg, 1987.

11. _ Error estimates for the $H_{0}^{1}(\Omega)$ and $L^{2}(\Omega)$ projections onto finite element spaces under weak regularity assumptions, Preprint, Chalmers University of Technology, Göteborg, 1988.

12. __ Basic error estimates for adaptive finite element methods for linear elliptic and parabolic problems, Numerical Methods and Applications (Sofia, 1989), pp. 141-147, Bulgar. Acad. Sci., Sofia, 1989.

13. K. Eriksson and C. Johnson, An adaptive finite element method for linear elliptic problems, Math. Comp. 50 (1988), 361-383.

14. _ Adaptive finite element methods for parabolic problems I: a linear model problem, SIAM J. Numer. Anal. 28 (1991), 43-77.

15. L. Gastaldi and R.H. Nochetto, Sharp maximum norm error estimates for general mixed finite element approximations to second order elliptic equations, RAIRO Modél. Math. Anal. Numér. 23 (1989), 103-128.

16. D. Gilbarg and N.S. Trudinger, Elliptic partial differential equations of second order, Springer-Verlag, Berlin and New York, 1983.

17. E. Giusti, Minimal surfaces and functions of bounded variation, Birkhäuser, Boston, 1984.

18. P. Grisvard, Elliptic problems in nonsmooth domains, Pitman, Boston, 1985.

19. C. Johnson, Adaptive finite element methods for diffusion and convection problems, Comp. Methods Appl. Mech. Engrg. 82 (1990), 301-322.

20. R.H. Nochetto, Removing the saturation assumption in a posteriori error analysis, Istit. Lombardo Accar. Sci. Lett. Rend. A (to appear).

21. R.H. Nochetto, M. Paolini, and C. Verdi, An adaptive finite element method for two-phase Stefan problems in two space dimensions. Part I: stability and error estimates, Math. Comp. 57 (1991), 73-108; Supplement, Math. Comp. 57 (1991), S1-S11. 
22. R. Rodriguez, Some remarks on Zienkiewicz-Zhu estimator, Numer. Methods Partial Differential Equations (to appear).

23. A. Schatz and L. Wahlbin, Maximum norm estimates in the finite element method on plane polygonal domains. Part 1, Math. Comp. 32 (1978), 73-109; Part 2, Refinements, Math. Comp. 33 (1979), 73-109.

24. R. Verfürth, A posteriori error estimators for the Stokes equations, Numer. Math. 55 (1989), 309-325.

25. O.C. Zienkiewicz and J.Z. Zhu, A simple error estimator and adaptive procedure for practical engineering analysis, Internat. J. Numer. Methods Engrg. 24 (1987), 337-357.

Department of Mathematics and Institute for Physical Science and Technology, University of Maryland, College Park, Maryland 20742

E-mail address: rhn@math.umd.edu 